\title{
COMPARISON MODULI SPACES OF RIEMANN SURFACES
}

\author{
ERIC SCHIPPERS AND WOLFGANG STAUBACH \\ Dedicated to the memory of Alexander Vasil'ev
}

\begin{abstract}
We define a kind of moduli space of nested surfaces and mappings, which we call a comparison moduli space. We review examples of such spaces in geometric function theory and modern Teichmüller theory, and illustrate how a wide range of phenomena in complex analysis are captured by this notion of moduli space. The paper includes a list of open problems in classical and modern function theory and Teichmüller theory ranging from general theoretical questions to specific technical problems.
\end{abstract}

\section{INTRODUCTION}

Theorems in complex analysis should be conformally invariant. In any branch of mathematics, one does not distinguish objects with respect to some equivalence; in complex analysis, this is conformal equivalence. Thus, if one alters all objects in the hypothesis of a theorem by applying a biholomorphism, then the theorem should apply to the new situation.

That is not to say that theorems which are not manifestly conformally invariant are not interesting. Indeed any pair of points on two distinct Riemann surfaces are contained in locally biholomorphic neighbourhoods by the Riemann mapping theorem, so local phenomena need not ever refer to conformal invariance. Furthermore we have powerful uniformization theorems at our disposal which reduce problems involving Riemann surfaces or mappings between them to canonical cases. This accounts for the fact that many deep results in complex analysis need not mention conformal invariance. However, it is still profitable to attempt to formulate - or reformulate - complex analytic results invariantly.

It is not always obvious how to do this. In the case of results about compact Riemann surfaces, it is straightforward. These Riemann surfaces are characterized by a finite set of conformal invariants, or by a finite-dimensional function space which is easily defined conformally invariantly, such as the vector space of holomorphic abelian differentials. However, things change radically when the Riemann surfaces have boundary curves. For example, the function spaces become infinite-dimensional, boundary values might enter their definition, and furthermore one must become concerned with boundary regularity of the biholomorphism. Thus many important complex analytic phenomena do not easily fit into the framework which is so successful for compact surfaces.

An example will illustrate the point. The universal Teichmüller space can be identified with the set of suitably normalized domains in the sphere bounded by quasicircles, and

Date: July 31, 2017.

Eric Schippers and Wolfgang Staubach are grateful for the financial support from the Wenner-Gren Foundations. Eric Schippers is also partially supported by the National Sciences and Engineering Research Council of Canada. 
can be thought of as the set of deformations of the complex structure of the unit disk. Consider the following reasonable objection to this definition, encountered occasionally by the authors: by the Riemann mapping theorem, these domains (or deformations) are all conformally equivalent. So why would one care about such a space? In other words, by conformal invariance, the universal Teichmüller space is of little interest. However, if one takes this objection too seriously, one will never find out that the universal Teichmüller space contains all Teichmüller spaces, or learn the beautiful geometric and analytic theory of quasidisks and quasiconformal mappings. Thus this naive approach to conformal invariance is not appropriate for quasiconformal Teichmüller theory.

How then do we introduce conformal invariance into this picture? This is done by taking into account the fact that the universal Teichmüller space involves two Riemann surfaces: the quasidisk and the sphere itself. Thus it is a moduli space of embeddings of disks into the sphere.

In this paper, we outline a general notion of moduli spaces of pairs of surfaces, or the closely related moduli spaces of mappings. We call them comparison moduli spaces. This notion of moduli spaces and the corresponding notion of conformal invariance capture a wide range of complex analytic phenomena. We show how comparison moduli spaces are present in classical geometric function theory, and along the way draw attention to some deep ideas of Z. Nehari and M. Schiffer which in our opinion have received too little attention. We also review some modern manifestations of the idea in quasiconformal Teichmüller theory and conformal field theory, with special attention to the so-called Weil-Petersson class Teichmüller space. The paper also contains a list of open problems.

In attempting to convey a unifying viewpoint, we have chosen to paint a broad rather than a specific picture. Thus, our choice of topics is selective; especially so when we are dealing with older topics. We have not attempted to make a survey of current or past research on each topic, and have confined ourselves to providing a few key references which should be viewed as entry points to the literature. However, since the Weil-Petersson class Teichmüller theory of non-compact surfaces is rather new, we made an exception for this case and gave a more comprehensive literature review.

The organization of this paper is as follows. In Section 2, we define and give examples of comparison moduli spaces and the corresponding notion of conformal invariance. In Section 3 we review examples of such moduli spaces in geometric function theory, in the work of Nehari and Schiffer. In Section 4 we give a brief overview of quasiconformal Teichmüller theory, and the rigged moduli space arising in conformal field theory. We describe the correspondence between these two, which also demonstrates that Teichmüller space is, up to a discontinuous group action, a comparison moduli space. Finally, in Section 5, we give an overview of new developments in Weil-Petersson Teichmüller theory. We also briefly list some of the applications of this new field.

Acknowledgements. The authors are grateful to David Radnell for a fruitful collaboration and valuable discussions through the years, and for his comments and suggestions concerning the initial draft of the manuscript. 


\section{Comparison moduli spaCeS}

By a comparison moduli space, we mean a moduli space of one of two types, which we now describe. Our purpose is to illustrate the similarity of a wide range of ideas, and how the notion of comparison moduli space is useful in capturing complex analytic phenomena. Our purpose is not to give a Bourbaki styled axiomatization. Although the definitions of the comparison moduli spaces can easily be made precise, this introduces unnecessary abstractions. Thus, rather than taking this approach, we give them informal but general definitions. Precise definitions are reserved for the many examples throughout the paper.

2.1. Moduli space of nested surfaces. Let $R_{1}$ and $R_{2}$ be Riemann surfaces such that $R_{2} \subseteq R_{1}$. We will also usually equip the nested surfaces with extra data $a, b, c, \ldots$ We assume that for any biholomorphism $g: S_{1} \rightarrow R_{1}$, there is an operation $g^{*}$ on the data denoted $a \mapsto g^{*}(a)$ which takes data on $\left(R_{1}, R_{2}\right)$ to data on $\left(S_{1}, S_{2}\right)$. For example, a might be a specified point in $S_{1}$ and $g^{*}(a)=g^{-1}(a)$, while $b$ might be a harmonic function on $S_{1} \backslash\{a\}$ and $g^{*} b=b \circ g$. This operation should be invertible and respect composition: that is, $\left(g_{1} \circ g_{2}\right)^{*}(a)=g_{2}^{*}\left(g_{1}^{*}(a)\right)$ and $\left(g^{-1}\right)^{*}=\left(g^{*}\right)^{-1}$. We call this operation the pull-back under $g$, and call the set $\left\{\left(R_{1}, R_{2}, a, b, c, \ldots\right)\right\}$ the configuration space $\mathfrak{C}$.

Two elements of the configuration space are equivalent

$$
\left(R_{1}, R_{2}, a, b, c, \ldots\right) \sim\left(S_{1}, S_{2}, A, B, C, \ldots\right)
$$

if and only if there is a biholomorphism $g: S_{1} \rightarrow R_{1}$ such that

$$
g\left(S_{2}\right)=R_{2}, \quad g^{*}(a)=A, \quad g^{*}(b)=B, \quad g^{*}(c)=C, \ldots .
$$

The moduli space of nested surfaces $\mathfrak{M}$ is the set of equivalence classes

$$
\mathfrak{M}=\mathfrak{C} / \sim \text {. }
$$

A conformal invariant is a function on the configuration space $I: \mathfrak{C} \rightarrow \mathbb{C}$ (or into $\mathbb{R}$ ) such that for any biholomorphic map $g: S_{1} \rightarrow R_{1}$ such that $g\left(S_{2}\right)=R_{2}$ one has

$$
I\left(R_{1}, R_{2}, a, b, c, \ldots\right)=I\left(S_{1}, S_{2}, g^{*}(a), g^{*}(b), g^{*}(c), \ldots\right) .
$$

Equivalently, it is a function on $\mathfrak{M}$.

Remark 2.1. The definition 2.1 can be generalized easily to allow for the possibility that $I$ is a differential of order $n$ on $R_{2}$ (that is, given in local coordinates by $h(z) d z^{n}$ ).

Finally, one might place restrictions on the Riemann surfaces (e.g. bounded by quasicircles, fixed genus) or the data (e.g. harmonic functions of finite Dirichlet energy, singularity of specified form at a point). It is required that these conditions are conformally invariant.

Example 2.1. Let $\mathfrak{C}=\left\{\left(R_{1}, R_{2}, z\right)\right\}$ such that $R_{1}$ and $R_{2}$ are hyperbolic Riemann surfaces, $R_{2} \subseteq R_{1}$ and $z \in R_{2}$. Let $\lambda_{i}(z)^{2}|d z|^{2}$ denote the hyperbolic metric on $R_{1}$ in any fixed local coordinate (with curvature normalized to -1 say). For a biholomorphism $g: S_{1} \rightarrow R_{1}$ define $g^{*}(z)=g^{-1}(z)$. Define

$$
I\left(R_{1}, R_{2}, z\right)=\lambda_{1}(z) / \lambda_{2}(z) .
$$

This is independent of the choice of coordinate and conformally invariant.

Another example of a conformal invariant is the following [62]. Defining $\Gamma_{i}=2 \frac{\partial}{\partial z} \log \lambda_{i}$ in some local coordinate, the quantity

$$
I_{2}\left(R_{1}, R_{2}, z\right)=\lambda_{3}^{-1}(z)\left|\Gamma_{2}(z)-\Gamma_{1}(z)\right|
$$


is independent of choice of coordinate and conformally invariant.

Example 2.2. Let $\mathfrak{C}=\left\{\left(R_{1}, R_{2}\right)\right\}$ where $R_{1}$ is a simply connected Riemann surface which is biholomorphic to the disk and $R_{2} \subseteq R_{1}$ is bounded by a quasicircle in $R_{1}$. The condition that $R_{2}$ is bounded by a quasicircle in $R_{1}$ is conformally invariant.

Example 2.3. Let $\mathfrak{C}=\left\{\left(R_{1}, R_{2}, h\right)\right\}$ where $R_{1}$ and $R_{2}$ are doubly connected Riemann surfaces (i.e. biholomorphic to $r<|z|<R$ for $0<r<R<\infty$ ), $R_{2} \subseteq R_{1}$ and $h$ is a harmonic function on $R_{1}$ of finite Dirichlet energy:

$$
\iint_{R_{1}} d h \wedge \overline{d h}<\infty
$$

These conditions are conformally invariant. For a biholomorphism $g: S_{1} \rightarrow R_{1}$ define $g^{*} h=h \circ g$. The quantity

$$
I\left(R_{1}, R_{2}, h\right)=\iint_{R_{1} \backslash R_{2}} d h \wedge \overline{d h}
$$

is a conformal invariant.

Example 2.4. Let $\mathfrak{C}=\left\{\left(R_{1}, R_{2}, a, b, c\right)\right\}$ where $R_{1}$ is a Riemann surface biholomorphic to the Riemann sphere, $R_{2} \subseteq R_{1}$ is bordered by a quasicircle in $R_{1}$, and $a, b, c$ are points on $\partial R_{2}$. For a biholomorphism $g: S_{1} \rightarrow R_{1}$ we define $g^{*}(a)=g^{-1}(a)$, and similarly for $b$ and $c$. The moduli space $\mathfrak{M}$ is then the universal Teichmüller space. This will be discussed in Section 4.2 ahead.

2.2. Moduli spaces of mappings. We consider also moduli spaces of mappings between Riemann surfaces. That is, we define a configuration space $\mathfrak{C}=\left\{\left(R_{0}, f, R, a, b, c, \ldots\right)\right\}$ where $R_{0}$ is a fixed Riemann surface, $R$ is a variable Riemann surface, $f: S \rightarrow R$ is an injective holomorphic map, and $a, b, c, \ldots$ are data as above. Pull-backs under biholomorphisms are defined as above. We define an equivalence relation $\left(R_{0}, f, R, a, b, c, \ldots\right) \sim\left(R_{0}, F, S, A, B, C, \ldots\right)$ if and only if there is a biholomorphism $g: S \rightarrow R$ such that $f=g \circ F, g^{*}(a)=A, g^{*}(b)=B$, $g^{*}(c)=C, \ldots$. The moduli space of mappings is

$$
\mathfrak{M}=\mathfrak{C} / \sim \text {. }
$$

A conformal invariant is a quantity $I$ on $\mathfrak{C}$ such that for any biholomorphism $g: S \rightarrow R$

$$
I\left(R_{0}, g \circ F, R, a, b, c, \ldots\right)=I\left(R_{0}, F, S, g^{*}(a), g^{*}(b), g^{*}(c), \ldots\right) .
$$

Equivalently, it is a function on $\mathfrak{M}$.

We give further examples. Denote the unit disk by $\mathbb{D}=\{z:|z|<1\}$, the complex plane by $\mathbb{C}$, and the Riemann sphere by $\overline{\mathbb{C}}$.

Example 2.5. Let $\mathfrak{C}=\{(\mathbb{D}, f, R, z)\}$ where $R$ is a simply connected Riemann surface conformally equivalent to $\mathbb{D}, f$ is an injective holomorphic mapping such that $f(0)=z$. Then $\mathfrak{M}$ is in one-to-one correspondence with the set of bounded univalent functions $f: \mathbb{D} \rightarrow \mathbb{D}$ such that $f(0)=0$.

Note that if we set $R_{1}=R$ and $R_{2}=f(\mathbb{D})$ then $\mathfrak{C}$ and $\mathfrak{M}$ corresponds to the comparison moduli spaces of Example 2.1, modulo an action by $\mathbb{S}^{1}$ (since the domain $f(\mathbb{D}$ ) does not uniquely determine $f$ ). Setting $z=0$ and $R=\mathbb{D}$ we can compute the conformal invariant $I_{2}$ explicitly in terms of $f$, namely $I_{2}(\mathbb{D}, f, R, z)=\left|f^{\prime \prime}(0) / f^{\prime}(0)^{2}\right|$. Since this is invariant 
under the $\mathbb{S}^{1}$-action $f(z) \mapsto e^{-i \theta} f\left(e^{i \theta} z\right)$, the invariant $I_{2}$ is well-defined on the configuration $\mathfrak{C}$ considered here. The general expression in terms of $z$ is more complicated.

Remark 2.2. The notion of comparison moduli spaces is required in order to formulate statements regarding higher-order derivatives of maps conformally invariantly [67].

Example 2.6. Let $\mathfrak{C}=\{(\mathbb{D}, f, R)\}$ where $R$ is conformally equivalent to the sphere $\overline{\mathbb{C}}, a, b, c$ are points in $R$, and $f: \mathbb{D} \rightarrow R$ is an injective holomorphic map with a quasiconformal extension to $R$. Then $\mathfrak{M}$ is easily seen to be in one-to-one correspondence with Example 2.4, and thus is the universal Teichmüller space. Other choices of normalization on $f$ can be imposed.

Example 2.7. Let $\mathfrak{C}=\left\{\left(\mathbb{D}, f_{1}, \ldots, f_{n}, R, p_{1}, \ldots, p_{n}\right)\right\}$ where $R$ is a compact Riemann surface of genus $g, p_{1}, \ldots, p_{n} \in R, f_{i}: \mathbb{D} \rightarrow R$ such that $f_{i}$ are one-to-one holomorphic maps on the closure of the disk cld , the closures of the images of $\mathbb{D}$ do not overlap, and $f_{i}(0)=p_{i}$. Then $\mathfrak{M}$ is a moduli space in conformal field theory due to D. Friedan and S. Shenker, G. Segal, and C. Vafa, see Y-Z. Huang [25] for references. The case where analyticity on cliD is weakened to quasiconformal extendibility will be considered at length in this paper.

As one can also see from these examples, given a moduli space of nested surfaces, there is a corresponding moduli space of mappings, if one adds the correct data.

\section{Some EXAmples in GeOMETRIC FUnCTION THEORY}

3.1. Nehari monotonicity theorems and generalizations. Classically, Riemann surfaces are characterized by their spaces of functions or differentials with specified singularities. The existence of functions or differentials with specified singularities is closely related to the Dirichlet problem on Riemann surfaces. In the case of compact surfaces, algebraic geometric techniques nearly (but not quite) eliminate the need to consider the Dirichlet problem. On the other hand, for nested surfaces with boundaries, there is not sufficient rigidity for these algebraic geometric techniques to determine the families of differentials, and the Dirichlet problem again takes centre stage.

Z. Nehari [38] defined a class of functionals on subdomains of Riemann surfaces, obtained from the Dirichlet integrals of harmonic functions of specified singularities. It is closely related to the so-called contour integral and area techniques in function theory. We will not review these connections since they can be found in other sources.

Nehari considered the comparison moduli space $\mathfrak{C}$ (he did not use the term), which in our terminology is given by $\mathfrak{C}=\left\{\left(R_{1}, R_{2}, z_{1}, z_{2}, \ldots, z_{n}, h\right)\right\}$ where $R_{1}$ and $R_{2}$ are Riemann surfaces, $R_{2} \subseteq R_{1}$ is bounded by analytic curves in the interior of $R_{1}, z_{1}, \ldots, z_{n} \in R_{2}$ and $h$ is a harmonic function on $R_{1}$ with singularities at $z_{i}$. He did not precisely specify the nature of the singularities of $h$, but in all examples they were such that $h$ was locally the real part of a meromorphic function with poles at $z_{i}$. If we define pull-back on points and the harmonic function in the obvious way, these conditions are conformally invariant and thus we obtain the moduli space $\mathfrak{M}$.

Below, we shall give a collection of monotonic functionals on $\mathfrak{M}$ defined by Nehari. We will also shorten the notation of the configuration space to $\left(R_{1}, R_{2}, h\right)$ (since the information of the location of the singularities are encoded in the domain of the harmonic function $h$ ). 
Definition 3.1 (Nehari functional). Let $R$ be a Riemann surface, and let $D$ be a subdomain of $R$ bounded by finitely many analytic curves. Let $h$ be a harmonic function with a finite number of isolated singularities all of which are in $D$. Let $q$ be the unique function on $D$ which is constant on each component of $\partial D$, such that $q+h$ is harmonic on $D$, and such that for any closed contour $\Gamma$ in $D$,

$$
\int_{\Gamma} \frac{\partial(q+h)}{\partial n} d s=0
$$

Here $n$ denotes the unit outward normal and $d s$ denotes the unit arc length. We define the "Nehari functional" as

$$
N(R, D, h)=\int_{\partial D} h \frac{\partial q}{\partial n} d s
$$

Remark 3.2. Although the outward normal is coordinate dependent, the expression $h \frac{\partial q}{\partial n} d s$ is not.

It is immediately evident that the Nehari functional is conformally invariant in the sense that if $g: R \rightarrow R^{\prime}$ is a biholomorphism of Riemann surfaces then $N(R, D, h)=N\left(R^{\prime}, g(D), h \circ\right.$ $\left.g^{-1}\right)$.

Theorem 3.3 (Nehari [38]). $N$ is a monotonic functional in the sense that whenever $D_{1} \subseteq$ $D_{2}$ it holds that $N\left(R, D_{2}, h\right) \leq N\left(R, D_{1}, h\right)$.

This follows from the Dirichlet principle. It can be generalized to collections of nonoverlapping domains.

Nehari showed that this concept unifies a large number of results in function theory, including the Grunsky inequalities, many coefficient estimates for univalent functions, and estimates on capacity. Diverse applications from the point of view of capacitance can also be found in the monograph of V. Dubinin [11].

We restrict now to the case that both domains are simply connected and hyperbolic. In 62, 63, E. Schippers obtained many estimates on conformal invariants involving bounded univalent functions from Nehari's method. The order of the estimates are determined by the order of the singularity. By Teichmüller's principle [27], extremal problems are in general associated with quadratic differentials (this principle was enunciated by J. A. Jenkins who attributes it to $\mathrm{O}$. Teichmüller). If the estimate involves $f^{(n)}(z)$ at a fixed point $z$, then the associated quadratic differential has a pole of order $n+1$ at $z$.

Thus, one is led to

(1) formulate Nehari's functional in terms of quadratic differentials.

(2) consider quadratic differentials with poles of arbitrary order.

In constructing this reformulation in terms of quadratic differentials, we restrict to the case that the outside domain $R$ is conformally equivalent to a disk. Since we are dealing with domains with a global coordinate, we will use the notation $Q(z) d z^{2}$ for quadratic differentials. In 66] E. Schippers showed that, by altering $h$, every Nehari functional is up to a constant equal to a Nehari functional such that the boundary of $R$ is a trajectory of the quadratic differential $Q(z) d z^{2}=h^{\prime}(z)^{2} d z^{2}$. Furthermore, this particular choice of $h$ is precisely the one so that $N(R, R, h)=0$.

Of course, this strongly suggests that the Nehari functional can be generalized to arbitrary quadratic differentials, not just those of the form $h^{\prime}(z)^{2} d z^{2}$. This can indeed be done by 
passing to a double cover $\pi: \tilde{R} \rightarrow R$ branched at odd-order poles and zeros [66, 67]. For any quadratic differential $Q(z) d z^{2}$ such that $\partial R$ is a trajectory, define

$$
m\left(R, D, Q(z) d z^{2}\right)=\int_{\partial \tilde{D}} h \frac{\partial q}{\partial n} d s
$$

where $\tilde{D}=\pi^{-1}(D), h=\operatorname{Re} \int \sqrt{Q(\pi(z)) \pi^{\prime}(z)^{2}} d z$, and $q$ is the unique harmonic function on $\tilde{D}$ such that $q=h$ on $\partial \tilde{D}$. We also assume that the boundary of $D$ is sufficiently regular, but the functional extends to the case that $D$ is bounded by a Jordan curve. Some effort is required to make this definition rigorous; one must deal with branch points, show that this integral is single-valued and independent of choice of branch of the square root, etc. Once all these issues are settled, one obtains the following result:

Theorem 3.4 ([66]). Let $R$ and $D$ be conformally equivalent to the unit disk, such that $D \subseteq R$, let $Q(z) d z^{2}$ be a quadratic differential such that $\partial R$ is a trajectory, all of whose poles are contained in $D$. Then the following statements hold:

(1) $m\left(R, D, Q(z) d z^{2}\right)$ is conformally invariant in the sense that if $g: R \rightarrow R^{\prime}$ is a conformal bijection and $g(D)=D^{\prime}$ then $m\left(R^{\prime}, D^{\prime}, Q(z) d z^{2}\right)=m\left(R, D, Q(g(z)) g^{\prime}(z)^{2} d z^{2}\right)$;

(2) if $D_{1} \subseteq D_{2}$ then $m\left(R, D_{1}, Q(z) d z^{2}\right) \leq m\left(R, D_{2}, Q(z) d z^{2}\right)$;

(3) for any $D, m\left(R, D, Q(z) d z^{2}\right) \leq 0$;

(4) if $m\left(R, D, Q(z) d z^{2}\right)=0$ then $D$ is $R$ minus arcs of trajectories of $Q(z) d z^{2}$;

(5) if $Q(z) d z^{2}=h^{\prime}(z)^{2} d z^{2}$ for some harmonic function $h$ with singularities, then $m\left(R, D, Q(z) d z^{2}\right)=(-1 / 2) N(R, D, h)$.

Remark 3.5. These invariants can be extended to domains slit by analytic arcs.

By choosing $R=\mathbb{D}$ and $D=f(\mathbb{D})$ for a univalent function $f$, many inequalities for bounded univalent functions follow [66, 67]. The functional associated with $Q(z) d z^{2}$ is in accordance with Teichmüller's principle. That is, if the quadratic differential has a pole of order $n+1$ at $z$, the $n$th derivative of the mapping function $f^{(n)}(z)$ arises in the functional.

We now pose the following problem:

Problem 3.1. Generalize this to the case that the inner domain is bordered by quasicircles.

The geometric motivation for this problem will be discussed ahead; see Problems 4.1, 4.3, and 4.4 .

The next problems are much harder, and are at the root of many problems in geometric function theory. In the remainder of the section, we restrict to the case of two nested simply connected hyperbolic Riemann surfaces (although it is clear that the problems have natural generalizations). By conformal invariance we may choose $R=\mathbb{D}$. We have that any conformal map from $\mathbb{D}$ into $\mathbb{D}$ which is admissible for a quadratic differential $Q(z) d z^{2}$ attains the upper bound of the functional $m\left(\mathbb{D}, f(\mathbb{D}), Q(z) d z^{2}\right)$.

Now suppose that we are given a functional in advance, which is not obviously of the form $m\left(\mathbb{D}, f(\mathbb{D}), Q(z) d z^{2}\right)$ - for example, a coefficient functional for bounded univalent functions. 
How does one choose a quadratic differential so that $m\left(\mathbb{D}, f(\mathbb{D}), Q(z) d z^{2}\right)$ is the desired functional? Thus we have two problems.

Problem 3.2. Let $\Phi_{Q(z) d z^{2}}(f)=m\left(\mathbb{D}, f(\mathbb{D}), Q(z) d z^{2}\right)$. Describe the map $Q(z) d z^{2} \mapsto \Phi_{Q(z) d z^{2}}$ algebraically.

Conversely we ask, in the same spirit,

Problem 3.3. Describe the set of functionals arising from quadratic differentials (say with a pole at the origin, and no other poles). Describe the inverse map $\Phi_{Q(z) d z^{2}} \mapsto Q(z) d z^{2}$.

There are many interpretations of "algebraically", of course. A satisfactory answer will almost certainly involve the Lie-theoretic properties of the classes of (sufficiently regular) bounded and unbounded univalent functions; see e.g. A. A. Kirillov and D. V. Yuri'ev [29], I. Markina, D. Prokhorov and A. Vasil'ev [33], and Schippers [64].

The heart of the problem is that the relation between quadratic differentials and functionals has been imprecise since its introduction by Schiffer. It is a hard problem to construct a method for producing inequalities. It is still harder to obtain an inequality which one decides on at the outset, no matter which of the existing methods is used. This problem has never been satisfactorily resolved, in spite of the many successes of the theory of extremal problems.

It has long been known that there is a correspondence between quadratic differentials, boundary points of function spaces, and extremals of functions. The following references could serve as a starting point: P. Duren [12, C. Pommerenke [41], A. Schaeffer and D. Spencer [55], and O. Tammi [73, 74]. Although there are many heuristic principles and concrete theorems, the precise relation has never been established. This may be partly because the proof of the Bieberbach conjecture by deBranges has had the unfortunate effect of diverting attention from the problem (unjustly, since the Bieberbach conjecture involves only a single point on the boundary).

A full exposition of these problems would take more space than we have here, so we will content ourselves by quoting an elegant result of A. Pfluger. Since this result strongly suggests that much more remains to be discovered, we will follow it with a few related problems.

Theorem 3.6 ([40]). Consider the class of mappings $f(z)=z+a_{2} z^{2}+a_{3} z^{3}+\cdots$ where $f: \mathbb{D} \rightarrow \mathbb{C}$ is holomorphic and one-to-one. For every $\lambda \in \mathbb{C} \backslash\{1\}$, there are precisely two mappings $f_{\lambda}(z)$ and $-f_{\lambda}(-z)$ maximizing the functional $f \mapsto \operatorname{Re}\left(a_{3}-\lambda a_{2}^{2}\right)$. For every $A \in \mathbb{C} \backslash\{0\}$, with the exception of the Koebe function, every function $f$ mapping onto the plane minus trajectories of the quadratic differential $(1+A w) w^{-4} d w^{2}$ is extremal for precisely one such functional.

The class of functions is closely related to the universal Teichmüller space. In fact, the set of such functions which are additionally supposed to be quasiconformally extendible can be identified with the Bers fibre space over the Teichmüller space [72], which is also a comparison moduli space. In some sense Pfluger's result gives information about the boundary of this moduli space. Some natural questions follow.

Problem 3.4. Does Pfluger's result hold for higher-order functionals? For example, is there an analogous result for functionals of the form $\operatorname{Re}\left(a_{4}+\lambda a_{3} a_{2}+\mu a_{2}^{3}\right)$ and quadratic differentials of the form $\left(\left(1+A w+B w^{2}\right) / w^{5}\right) d w^{2}$ ? 
The form of the functional is obtained by demanding that the expression be homogeneous with respect to the transformation $f(z) \mapsto e^{-i \theta} f\left(e^{i \theta} z\right)$, while the form of the quadratic differential can be obtained using Schiffer's variational theorem to differentiate the functional [41, Chapter 7].

Similarly, we can ask whether Pfluger's results hold in some sense for the comparison moduli space considered throughout this section:

Problem 3.5. Is there a version of Pfluger's result for bounded one-to-one holomorphic mappings $f: \mathbb{D} \rightarrow \mathbb{D}$ such that $f(0)=0$ ?

We observe that Pfluger's result was obtained by combining Schiffer variation with J. Jenkins' quadratic differential/extremal metric methods. Possibly the generalization of Nehari's results to quadratic differentials might shed further light on these questions.

Finally, we state some general problems on the relation to Schiffer variation. The Schiffer variational method (in its various forms) says that for various classes of conformal maps, the extremal map of a (reasonably regular) fixed functional maps onto the target domain minus trajectories of a quadratic differential [12, 41. However, the quadratic differential is not precisely determined and depends to some extent on the unknown extremal function. In some sense, the Schiffer variational method produces the functional derivative of a functional at an extremal and relates it to the quadratic differential (see [41, Theorem 7.4]).

In the case of the invariants (3.1), the extremal condition suggests that the quadratic differential obtained by Schiffer variation equals the original quadratic differential. We are thus led to ask:

Problem 3.6. What is the relation between the functional derivative of $m\left(\mathbb{D}, f(\mathbb{D}), Q(z) d z^{2}\right)$ and the quadratic differential $Q(z) d z^{2}$ ?

By using the Loewner method to obtain functional derivatives, it was shown in [65] that in the case of Nehari functionals (that is, for quadratic differentials of the form $h^{\prime}(z)^{2} d z^{2}$ ) with poles only at the origin, the functional derivative at an extremal is the pull-back of the original quadratic differential under the extremal map. We conjecture that this holds in much greater generality, and also ask whether a stronger statement can be made.

3.2. A few remarks on the extremal metric method. J. A. Jenkins' extremal metrics method associates functionals to quadratic differentials [27]. This method is based on lengtharea inequalities of H. Grötzsch [21] and Teichmüller [76], which grew into the theory of extremal length. A modern exposition can be found in the monograph of A. Vasiliev [77]. According to Jenkins [27], one can interpret these estimates as involving reduced modules; see H. G. Schmidt 68] for an enlightening exposition in special cases. However, in practice, this statement is difficult to make precise except for quadratic differentials of order two or lower (see e.g. Dubinin [11, Chapter 6]). Also, Jenkins' method does not manifestly produce conformally invariant functionals for quadratic differentials of order strictly greater than two. Thus one is led to the following problems:

Problem 3.7. Find a systematic definition of conformally invariant reduced modules in terms of extremal length of curve families, which produces estimates on arbitrary-order derivatives of conformal maps.

This is deliberately imprecise, since it has not even been done in the case of bounded univalent maps. Here is a more precise formulation in that special case. 
Problem 3.8. Let $D_{2} \subseteq D_{1}$ be simply connected domains and let $z$ be a point in $D_{2}$. Define conformally invariant reduced modules of curve families which for $D_{1}=\mathbb{D}$ and $z=0$, explicitly involving the $n$th derivative of the conformal map $f: \mathbb{D} \rightarrow D_{1}$ taking 0 to 0 . Prove estimates on these modules using extremal length methods of Grötzsch/Teichmüller/Jenkins.

The boundaries of the domains could be chosen as regular as necessary, although we note in passing that the quasiconformal invariance of the notion of prime ends should allow a quite general formulation.

These reduced modules should be associated with quadratic differentials through their curve families.

Problem 3.9. Systematically associate a conformally invariant reduced module to each quadratic differential (with poles of arbitrary order).

Again, this can be formulated for various classes of maps or pairs of domains, but it would be of great interest even for bounded univalent maps/simply connected domains.

In some sense Theorem 3.4 of the first author is an answer to Problem 3.9, but with Nehari's Dirichlet energy technique replacing extremal length. Now by a theorem of A. Beurling, the Dirichlet energy of a mixed Dirichlet/Neumann boundary value problem is the reciprocal of the extremal length of a curve family [5]. This immediately leads to the following problem.

Problem 3.10. Formulate and prove a generalization of Beurling's theorem for harmonic functions with singularities.

This problem was posed earlier in [63], before the discovery of Theorem 3.4. The answer to this problem will certainly involve the generalized Nehari invariants (3.1) and Jenkins' method of quadratic differentials.

3.3. Schiffer comparison theory of domains. Another form of the comparison moduli space idea is Schiffer's comparison theory of nested domains, where one compares the kernel functions of two domains $E$ and $\mathcal{E}$ where $E \subset \mathcal{E}$. The ideas go back to his foundational paper with S. Bergman [6] and his earlier paper [56]. Those papers deal with the special case $\mathbb{C}$; in this case the importance of the role of the outer domain is not obvious. The comparison theory of kernels on nested domains was principally investigated by Schiffer [60, 61, so we will attach his name to it. Schiffer's theory reached its final published form in his appendix to the book of R. Courant [8]. This section draws mainly on that source. We add some simple but important observations on conformal invariance.

Let $E$ be a planar domain with Green's function $g$. Define two kernel functions as follows.

Definition 3.7. The Bergman kernel of a domain $E$ with Green's function $g$ is

The Schiffer kernel of $E$ is

$$
K(w, z)=-\frac{2}{\pi} \frac{\partial^{2} g}{\partial \bar{w} \partial z}
$$

$$
L(w, z)=-\frac{2}{\pi} \frac{\partial^{2} g}{\partial w \partial z}
$$

The first appearance of the $L$-kernel is to our knowledge [56], hence we call it the Schiffer kernel. Clearly

$$
L(z, w)=L(w, z)
$$


and

$$
K(z, w)=\overline{K(w, z)}
$$

These kernels are conformally invariant in the sense that if $g: E \rightarrow E^{\prime}$ is a conformal bijection then

$$
L_{E^{\prime}}(g(w), g(z)) g^{\prime}(w) g^{\prime}(z)=L_{E}(w, z) \text { and } K_{E^{\prime}}(g(w), g(z)) \overline{g^{\prime}(w)} g^{\prime}(z)=K_{E}(w, z) .
$$

Strictly speaking it is best to view the kernel functions as differentials $L(w, z) d w d z$ and $K(w, z) d \bar{w} d z$.

We will define the Bergman and Schiffer kernels of the plane as follows.

Definition 3.8. The Bergman kernel of $\mathbb{C}$ is defined to be $K(z, w)=0$. The Schiffer kernel of $\mathbb{C}$ is

$$
L_{\mathbb{C}}(z, w)=\frac{1}{\pi} \frac{1}{(z-w)^{2}}
$$

These definitions can be motivated by computing the Schiffer kernel of the disk of radius $r$ and letting $r \rightarrow \infty$. Another motivation is that with that definition, the identities and inequalities continue to hold in the case that $\mathcal{E}=\mathbb{C}$.

Next we give two important properties of the kernel functions. Denote the Bergman space of a domain $E$ by

$$
A_{1}^{2}(E)=\left\{h: E \rightarrow \mathbb{C} \text { holomorphic }: \iint_{E}|h|^{2} d A\right\}<\infty .
$$

Remark 3.9. The Bergman space is best viewed as a space of one-differentials, but for now we view it as a function space to be consistent with the majority of the function theory literature.

Proposition 3.10. Let $f \in A_{1}^{2}(E)$. Then

$$
\iint_{E} K(\zeta, \eta) f(\eta) d A_{\eta}=f(\zeta)
$$

and

$$
\iint_{E} L(\zeta, \eta) \overline{f(\eta)} d A_{\eta}=0
$$

Let $E, E^{c}$ and $\mathcal{E}$ be domains bounded by finitely many smooth curves, satisfying $E \subset \mathcal{E}$ and $E^{\mathrm{c}} \subset \mathcal{E}$, in such a way that $\mathcal{E}$ is the union of $E$ and $E^{\mathrm{c}}$ together with their shared boundary curves. Note that $E^{\mathrm{c}}$ is not the literal complement in $\mathcal{E}$ but rather its interior. Denote by $E \bigsqcup E^{c}$ the disjoint union of $E$ and $E^{c}$. The inner products on each domain will be denoted $(,)_{E}$ etc.

Schiffer considered integral operators naturally associated with a configuration of nested domains. Let $K, K^{\mathrm{c}}$ and $\mathcal{K}$ be the Bergman kernels of $E, E^{\mathrm{c}}$ and $\mathcal{E}$ respectively. Similarly, let $L, L^{\mathrm{c}}$ and $\mathcal{L}$ be the Schiffer L-kernels associated with $E, E^{\mathrm{c}}$ and $\mathcal{E}$.

\section{Definition 3.11.}

$$
\begin{aligned}
T_{E, \mathcal{E}}^{1}: A_{1}^{2}(E) & \rightarrow A_{1}^{2}(\mathcal{E}) \\
f & \mapsto \iint_{E} \mathcal{K}(\cdot, w) f(w) d A_{w}
\end{aligned}
$$


Next we consider the operator associated with the Schiffer $L$-kernel.

Definition 3.12.

$$
\begin{aligned}
T_{E, \mathcal{E}}^{2}: \overline{A_{1}^{2}(E)} & \rightarrow A_{1}^{2}\left(E \sqcup E^{\mathrm{c}}\right) \\
\bar{f} & \mapsto \iint_{E} \mathcal{L}(\cdot, w) \overline{f(w)} d A_{w} .
\end{aligned}
$$

Here the inner product on $A_{1}^{2}\left(E \sqcup E^{\mathrm{c}}\right)$ is of course

$$
\left(h_{1}, h_{2}\right)_{E \sqcup E^{\mathrm{c}}}=\iint_{E \sqcup E^{\mathrm{c}}} h_{1} \overline{h_{2}} .
$$

Remark 3.13. Schiffer defines the operator $T_{E, \mathcal{E}}^{2}$ as a map from $A_{1}^{2}(E)$ into $A_{1}^{2}\left(E \sqcup E^{\mathrm{c}}\right)$. This is not convenient, since with that convention the operator is complex anti-linear.

There is a relation between $T_{E, \mathcal{E}}^{1}$ and $T_{E, \mathcal{E}}^{2}$.

Theorem 3.14. For all $f, g \in \overline{A_{1}^{2}(E)}$,

$$
\left(T_{E, \mathcal{E}}^{2} g, T_{E, \mathcal{E}}^{2} f\right)_{E \sqcup E^{\mathrm{c}}}=(g, f)_{E}-\left(T_{E, \mathcal{E}}^{1} g, T_{E, \mathcal{E}}^{1} f\right)_{E \sqcup E^{\mathrm{c}}}
$$

Thus

$$
T_{E, \mathcal{E}}^{2} T_{E, \mathcal{E}}^{2}=I-T_{E, \mathcal{E}}^{1} T_{E, \mathcal{E}}^{1}
$$

Theorem 3.14 immediately implies the bounded Grunsky inequalities (in Schiffer's form) for the $T$ operator.

Corollary 3.15. For $f \in A_{1}^{2}(E)$ one has that

$$
\left\|T_{E, \mathcal{E}}^{2} f\right\|_{E}^{2}=\|f\|_{E}^{2}-\left\|T_{E, \mathcal{E}}^{1} f\right\|_{E \cup E^{\mathrm{c}}}^{2}-\left\|T_{E, \mathcal{E}}^{2} f\right\|_{E^{\mathrm{c}}}^{2} .
$$

In particular,

$$
\left\|T_{E, \mathcal{E}}^{2} f\right\|_{E^{\mathrm{c}}} \leq\|f\|_{E}
$$

Remark 3.16. In the case that $E$ is simply connected, the classical Grunsky inequalities can be obtained by setting $E=g(\mathbb{D})$ for some conformal map $g, \mathcal{E}=\mathbb{C}$ and $f$ to be a polynomial in $1 / z$ in the above corollary. This can be found in Bergman-Schiffer [6, Section 9].

In Schiffer's comparison theory discussed above, one can identify a comparison moduli space as follows. We set $\mathcal{C}=\left\{(\mathcal{E}, E, h): h \in A_{1}^{2}(E)\right\}$, where as before $E \subseteq \mathcal{E}$ are planar domains. Let $g: \mathcal{E} \rightarrow \mathcal{E}^{\prime}$ be a bijective holomorphic map. Define the pull-back by

$$
\begin{aligned}
g^{*}: A_{1}^{2}\left(E^{\prime}\right) & \rightarrow A_{1}^{2}(E) \\
h & \mapsto h \circ g \cdot g^{\prime}
\end{aligned}
$$

(cf Remark 3.9) and similarly $g^{*} \bar{h}=\overline{h \circ g \cdot g^{\prime}}$. Then we have

Proposition 3.17. The operators $T_{1}$ and $T_{2}$ are conformally invariant in the following sense: If $g$ is a one-to-one holomorphic map from $E$ onto $E^{\prime}$, then for all $h \in A_{1}^{2}\left(E^{\prime}\right)$

$$
T_{E, \mathcal{E}}^{1}\left(g^{*} h\right)=g^{*} T_{E^{\prime}, \mathcal{E}^{\prime}}^{1}(h) \quad \text { and } \quad T_{E, \mathcal{E}}^{2}\left(g^{*} \bar{h}\right)=g^{*} T_{E^{\prime}, \mathcal{E}^{\prime}}^{2}(\bar{h}) .
$$


Thus if we define

$$
I^{1}(\mathcal{E}, E, h)=\left\|T_{E, \mathcal{E}}^{1} h\right\|_{A_{1}^{2}(E)} \quad \text { and } \quad I^{2}(\mathcal{E}, E, h)=\left\|T_{E, \mathcal{E}}^{2} \bar{h}\right\|_{A_{1}^{2}(E)}
$$

we obtain conformal invariants on $\mathfrak{C}$. Similarly the norms of $T_{E, \mathcal{E}}^{i}$ over $A_{1}^{2}\left(E^{\mathrm{c}}\right)$ are conformally invariant.

Remark 3.16] shows that it is possible to derive coefficient inequalities from Corollary 3.15. We summarize the familiar procedure [12, 41] in this notation. Setting $\mathcal{E}=\mathbb{C}$ and $E=g(\mathbb{D})$ for a univalent function $g: \mathbb{D} \rightarrow \mathbb{C}$, it is possible to write the operators $T_{g(\mathbb{D}), \mathbb{C}}^{2}$ and $T_{g(\mathbb{D}), \mathbb{C}}^{1}$ in terms of the coefficients of $g$. Assuming that $g(0)=0$, for every choice of test function $h$ which is polynomial in $1 / z$, one obtains a distinct inequality from Corollary 3.15. The same can be done for bounded univalent functions by replacing $\mathbb{C}$ with $\mathbb{D}$. The inequalities derived in this manner are conformally invariant.

Remark 3.18. The similarity to the Nehari-type invariants is of course not a coincidence.

Some generalizations of Schiffer's comparison theory to Riemann surfaces can be found in M. Schiffer and D. Spencer [57].

3.4. Fredholm determinant and Fredholm eigenvalues. The connection between the so-called Fredholm eigenvalues and Grunsky matrices was first observed by Schiffer, see e.g. [59]. One of Schiffer's accomplishments was to place Fredholm's real integral equations of potential theory in the complex setting. We draw on his insights freely here without justification, and refer the interested reader to [58, 59].

Given a domain $D$ in the plane, bounded by finitely many $C^{3}$ Jordan curves, consider the operator defined for $f \in A_{1}^{2}(D)$ by

$$
T_{D} f:=\left.\left(T_{D, \mathbb{C}}^{2} f\right)\right|_{D}
$$

where $T^{2}$ is the operator defined in Definition 3.12. The operator $T_{D}$ is a bounded operator from $\overline{A_{1}^{2}(D)}$ to $A_{1}^{2}(D)$. We note that this can even be extended to disconnected domains [58].

Moreover $T_{D}$ is a Hilbert-Schmidt operator. This yields that $T_{D} \overline{T_{D}}$ is a positive trace class operator, to which the spectral theorem is applicable $\left(T_{D} \overline{T_{D}}\right.$ is compact and self-adjoint). According to this theorem, there is an orthogonal basis $f_{n}$ of eigenfunctions of $T_{D} \overline{T_{D}}$ with non-negative eigenvalues $\lambda_{n}^{2}$. The non-zero $\lambda_{n}$ are called the Fredholm eigenvalues of $D$. The reader should however observe that this is not standard in the literature, as some authors refer to $1 / \lambda_{n}$ as the Fredholm eigenvalues. Now since $T_{D} \overline{T_{D}}$ is a trace class operator, one can define the Fredholm determinant of the domain $D$ as

$$
\Delta_{D}:=\operatorname{det}\left(I-T_{D} \overline{T_{D}}\right)=\prod_{n}\left(1-\lambda_{n}^{2}\right)
$$

Using variational techniques, Schiffer showed that the Fredholm determinant is conformally invariant in the sense of (2.1). More precisely, let $D_{1}, \ldots, D_{n}$ be a collection of bounded simply connected domains in $D$, each bounded by a $C^{3}$ Jordan curve. Let $E$ be the interior of the complement of $D_{1} \cup \cdots \cup D_{n}$ in $\mathbb{C}$. Fix an $l \in\{1, \ldots, n\}$ and let $\mathcal{E}=\mathbb{C} \backslash D_{l}$. Let 
$\mathfrak{C}=\{(\mathcal{E}, E)\}$ where $E$ and $\mathcal{E}$ are of the above form. In that case, the quantity

$$
I(\mathcal{E}, E)=\frac{\Delta_{\mathcal{E}}}{\Delta_{E}}
$$

is a conformal invariant in the sense of (2.1). Observe that this striking invariance property must involve pairs of domains, and therefore is a result regarding comparison moduli spaces.

Remark 3.19. Although we do not doubt the validity of this result, Schiffer's proof is rather heuristic, since it infers invariance under all conformal maps from invariance under a specific set of variations.

In the case that $D$ is simply connected, $T_{D}$ is a version of the Grunsky operator, as we have observed above. By work of L. Takhajan and L.-P. Teo [72], the Fredholm determinant relates to the Weil-Petersson metric. We will re-visit this in Section 5.4 ahead.

\section{TeichmülleR SPACE AS A COMPARISON MOdUli SPACE}

4.1. Spaces of non-overlapping maps and the rigged moduli space. Sets of maps with non-overlapping images are often considered in geometric function theory. In the general case of the sphere with $n$ non-overlapping maps, these are sometimes called the "GoluzinLebedev class" [19]. As we saw in Example [2.7, sets of non-overlapping conformal maps appear in conformal field theory. We will be concerned with the case that the mappings are quasiconformally extendible and the closures do not intersect.

Let

$$
A_{1}^{\infty}(\mathbb{D})=\left\{f: \mathbb{D} \rightarrow \mathbb{C} \text { holomorphic }: \sup _{z \in \mathbb{D}}\left(1-|z|^{2}\right)|f(z)|<\infty\right\}
$$

and recall that $A_{1}^{2}(\mathbb{D})$ is the Bergman space on the disk.

Definition 4.1. Let $\mathcal{O}^{\text {qc }}$ denote the set of maps $f: \mathbb{D} \rightarrow \mathbb{C}$ such that $f$ is an injective holomorphic map, $f(0)=0$ and $f$ is quasiconformally extendible to a neighbourhood of clDD. Let $\mathcal{O}_{\mathrm{WP}}^{\mathrm{qc}}=\left\{f \in \mathcal{O}^{\mathrm{qc}}: f^{\prime \prime} / f^{\prime} \in A_{1}^{2}(\mathbb{D})\right\}$.

Here "cl" denotes closure, and "WP" stands for Weil-Petersson. The terminology and motivation for this definition, as well as a review of the literature, will be given in Section 5 ahead.

Definition 4.2. Let $\Sigma$ be a Riemann surface of finite genus with $n$ ordered punctures $\left(p_{1}, \ldots, p_{n}\right) . \mathcal{O}^{\mathrm{qc}}(\Sigma)$ is the set of $n$-tuples of one-to-one holomorphic mappings $\left(f_{1}, \ldots, f_{n}\right)$ such that

(1) $f_{i}: \mathbb{D} \rightarrow \Sigma$ are one-to-one, holomorphic for $i=1, \ldots, n$

(2) $f_{i}(0)=p_{i}$

(3) $f_{i}$ has a quasiconformal extension to a neighbourhood of the closure of $\mathbb{D}$ for all $i$

(4) $f_{i}(\mathrm{cl} \mathbb{D}) \cap f_{j}(\mathrm{cl} \mathbb{D})$ is empty whenever $i \neq j$.

This defines a configuration space of mappings in the sense of Section 2.2. We will call such an $n$-tuple a "rigging" of $\Sigma$.

Observe also that $\left(f_{1}, \ldots, f_{n}\right) \in \mathcal{O}^{\mathrm{qc}}(\Sigma)$ if and only if there is at least one $n$-tuple of local coordinates $\left(\zeta_{1}, \ldots, \zeta_{n}\right)$ on open neighbourhoods $U_{i}$ of $\operatorname{cl} f(\mathbb{D})$ such that $\zeta_{i} \circ f_{i} \in \mathcal{O}^{\mathrm{qc}}$ for $i=1, \ldots, n$. We call such a chart an $n$-chart. If the condition holds for one $n$ chart, then it holds for every $n$ chart so that each $U_{i}$ contains $\operatorname{cl} f_{i}(\mathbb{D})=f_{i}(\mathrm{cl} D)$.

Radnell and Schippers showed that the set of riggings is a complex Banach manifold. 
Theorem 4.3 ([44]). Let $\Sigma$ be a Riemann surface with $n$ punctures. $\mathcal{O}^{\mathrm{qc}}(\Sigma)$ is a complex Banach manifold locally modelled on $\bigoplus^{n}\left(\mathbb{C} \oplus A_{1}^{\infty}(\mathbb{D})\right)$.

The charts of this manifold are

$$
\left(f_{1}, \ldots, f_{n}\right) \mapsto\left(\left(\zeta_{1} \circ f_{1}\right)^{\prime}(0), \frac{\left(\zeta_{1} \circ f_{1}\right)^{\prime \prime}}{\left(\zeta_{1} \circ f_{1}\right)^{\prime}}, \ldots,\left(\zeta_{n} \circ f_{n}\right)^{\prime}(0), \frac{\left(\zeta_{n} \circ f_{n}\right)^{\prime \prime}}{\left(\zeta_{n} \circ f_{n}\right)^{\prime}}\right)
$$

for some $n$-chart $\left(\zeta_{1}, \ldots, \zeta_{n}\right)$.

Similarly we have the Weil-Petersson class riggings.

Definition 4.4. Let $\Sigma$ be a Riemann surface with $n$ punctures. $\mathcal{O}_{\mathrm{WP}}^{\mathrm{qc}}(\Sigma)$ is the set of $\left(f_{1}, \ldots, f_{n}\right) \in \mathcal{O}^{\mathrm{qc}}(\Sigma)$ such that there is an $n$-chart such that $\zeta_{i} \circ f_{i} \in \mathcal{O}_{\mathrm{WP}}^{\mathrm{qc}}$.

Again, this defines a configuration space of mappings in the sense of (2.2).

Analogously to Theorem 4.3, Radnell, Schippers and Staubach showed that $\mathcal{O}_{\mathrm{WP}}^{\mathrm{qc}}(\Sigma)$ is a complex Hilbert manifold.

Theorem 4.5 ([48]). Let $\Sigma$ be a Riemann surface of finite genus with $n$ ordered punctures. $\mathcal{O}_{\mathrm{WP}}^{\mathrm{qc}}(\Sigma)$ is a complex Hilbert manifold locally modelled on $\bigoplus^{n}\left(\mathbb{C} \oplus A_{1}^{2}(\mathbb{D})\right)$.

The charts are obtained in the same way as for $\mathcal{O}^{\mathrm{qc}}(\Sigma)$.

We now define the rigged moduli space, which appears in conformal field theory and is closely related to the theory of vertex operator algebras [25].

Definition 4.6. The quasiconformally rigged moduli space is the set of equivalence classes

$$
\widetilde{\mathcal{M}}(g, n)=\{(R, \mathbf{f})\} / \sim
$$

where $R$ is a Riemann surface of genus $g$ with $n$ punctures, $\mathbf{f} \in \mathcal{O}^{\mathrm{qc}}(R)$ and the equivalence relation is defined by $(R, \mathbf{f}) \sim(S, \mathbf{g})$ if and only if there is a biholomorphism $\sigma: R \rightarrow S$ such that $\sigma \circ \mathbf{f}=\mathbf{g}$. The Weil-Petersson class rigged moduli space is the moduli space $\widetilde{\mathcal{M}}_{\mathrm{WP}}(g, n)$ defined as above with $\mathcal{O}^{\mathrm{qc}}(R)$ replaced with $\mathcal{O}_{\mathrm{WP}}^{\mathrm{qc}}(R)$.

By $\sigma \circ \mathbf{f}=\mathbf{g}$ we mean that $\sigma \circ f_{i}=g_{i}$ for $i=1, \ldots, n$ where $\mathbf{f}=\left(f_{1}, \ldots, f_{n}\right)$ and $\mathbf{g}=\left(g_{1}, \ldots, g_{n}\right)$.

In two-dimensional conformal field theory, various analytic choices for the riggings exist. We will see in the next section that the choices above lead to deep connections with Teichmüller theory.

A problem of immediate interest is the following:

Problem 4.1. Extend the invariants (3.1) to functions on $\widetilde{\mathcal{M}}(g, n)$; that is, to configuration spaces of maps into Riemann surfaces whose images are bounded by quasicircles.

This problem requires some clarification. The extension to Riemann surfaces was already accomplished by Nehari [38] for harmonic functions, and the methods of Schippers [66, 67] for quadratic differentials extend without difficulty to fairly arbitrary Riemann surfaces. The main issue is analytic, and amounts to extending the functionals to Riemann surfaces bordered by quasicircles. However, although the functionals can be extended continuously from maps with analytic boundaries to roughly bounded ones by exhaustion, it is highly desirable to have a natural definition which does not require special pleading. This could perhaps be done by replacing the contour integrals with appropriate integrals with respect to harmonic measure on the boundary of each quasicircle. 
A closely related problem is the following. Since the extremals of the functionals map onto $R$ minus trajectories of the quadratic differential, we ask

Problem 4.2. Can the rigged moduli space be endowed with a boundary which includes riggings that are maps onto $R$ minus trajectories of the quadratic differential? This must be done in such a way that the functionals extend continuously to this boundary.

4.2. A brief primer on quasiconformal Teichmüller theory. We give an overview of quasiconformal Teichmüller theory; for details see O. Lehto [31], S. Nag [34], or J. Hubbard [26]. First we define the Teichmüller space of a Riemann surface. Let $\Sigma$ be a Riemann surface whose universal cover is the disk. Given another Riemann surface $\Sigma_{1}$, a marking of this surface by $\Sigma$ is a quasiconformal map $f: \Sigma \rightarrow \Sigma_{1}$. Note that in particular this implies that $\Sigma_{1}$ is quasiconformally equivalent to $\Sigma$ (and thus in particular they are homeomorphic). We will denote marked Riemann surfaces by triples $\left(\Sigma, f, \Sigma_{1}\right)$.

We say that two quasiconformally equivalent marked Riemann surfaces are Teichmüller equivalent

$$
\left(\Sigma, f_{1}, \Sigma_{1}\right) \sim\left(\Sigma, f_{2}, \Sigma_{2}\right)
$$

if there is a biholomorphism $\sigma: \Sigma_{1} \rightarrow \Sigma_{2}$ such that $f_{2}^{-1} \circ \sigma \circ f_{1}$ is homotopic to the identity rel boundary. The term "rel boundary" means that the homotopy fixes the boundary pointwise. Making the meaning of boundary and homotopy rel boundary precise requires some effort in terms of the lift to the universal cover; we refer the readers to [26, 31] for a thorough treatment. The Teichmüller space can now be defined as follows.

Definition 4.7. Let $\Sigma$ be a fixed Riemann surface. The Teichmüller space of a Riemann surface $\Sigma$ is the set of equivalence classes

$$
T(\Sigma)=\left\{\left(\Sigma, f, \Sigma_{1}\right)\right\} / \sim
$$

where $f: \Sigma \rightarrow \Sigma_{1}$ is a quasiconformal marking of a Riemann surface $\Sigma_{1}$ and $\sim$ denotes Teichmüller equivalence. Denote equivalence classes by $\left[\Sigma, f, \Sigma_{1}\right]$.

An equivalent definition is given in terms of Beltrami differentials. A ( $-1,1)$-differential is one which is given in a local coordinate $z$ by

$$
\mu=h(z) \frac{d \bar{z}}{d z}
$$

for a Lebesgue-measurable complex-valued function $h$, and which transforms under a change of coordinate $z=g(w)$ by

$$
\tilde{h}(w)=h(g(w)) \frac{\overline{g^{\prime}(w)}}{g^{\prime}(w)} ; \quad \text { that is } \quad h(z) \frac{d \bar{z}}{d z}=\tilde{h}(w) \frac{d \bar{w}}{d w} .
$$

It is evident from the transformation rule that the essential supremum of a Beltrami differential is well-defined. For a Riemann surface $\Sigma$ define

$$
L_{-1,1}^{\infty}(\Sigma)_{1}=\left\{\text { measurable }(-1,1) \text { differentials } \mu \text { on } \Sigma:\|\mu\|_{\infty}<1\right\} .
$$

Given any $\mu \in L_{-1,1}^{\infty}(\Sigma)_{1}$ there is a quasiconformal solution $f$ to the Beltrami differential equation

$$
\mu=\frac{\bar{\partial} f}{\partial f}
$$


which is unique up to post-composition by a conformal map. We call $\mu$ in (4.2) the Beltrami differential of $f$. We say two Beltrami differentials $\mu, \nu$ are Teichmüller equivalent $\mu \sim \nu$ iff the corresponding solutions $\left[\Sigma, f_{\mu}, \Sigma_{\mu}\right]$ and $\left[\Sigma, f_{\nu}, \Sigma_{\nu}\right]$ are Teichmüller equivalent in the sense of Definition 4.7. The Teichmüller space can thus be identified with

$$
T(\Sigma)=\left\{\mu \in L_{-1,1}^{\infty}(\Sigma)_{1}\right\} / \sim
$$

via the map

$$
\left[\Sigma, f, \Sigma_{1}\right] \mapsto[\mu]
$$

In this paper, we restrict our attention to two possible kinds of Riemann surfaces: those of genus $g$ with $n$ punctures, and those of genus $g$ and $n$ boundary curves homeomorphic to the circle. In the border case we assume that the boundary curves are borders in the sense of L. Ahlfors and L. Sario [1]. We refer to these surfaces as punctured Riemann surfaces of type $(g, n)$ or bordered surfaces of type $(g, n)$.

We now describe the universal Teichmüller space, and in doing so fill out the details of Examples 2.4 and 2.6. Let $\mathbb{D}^{*}=\{z:|z|>1\} \cup\{\infty\}$. The universal Teichmüller space $T\left(\mathbb{D}^{*}\right)$ can be represented as follows. For a given quasiconformal marking $\left(\mathbb{D}^{*}, f, \Omega\right)$ we let $\hat{f}: \overline{\mathbb{C}} \rightarrow \overline{\mathbb{C}}$ be the quasiconformal map of the Riemann sphere which is conformal on $\mathbb{D}$ and whose Beltrami differential equals that of $f$ on $\mathbb{D}^{*}$. That is,

$$
\frac{f_{\bar{z}}}{f_{z}}=\frac{\hat{f}_{\bar{z}}}{\hat{f}_{z}} \text { a.e. on } \mathbb{D}^{*} \text {. }
$$

The resulting map $\hat{f}$ is unique up to post-composition with a Möbius transformation (and is traditionally specified through three normalizations). Stated in terms of the rigged moduli space, we have that the map

$$
\begin{aligned}
\Phi: T\left(\mathbb{D}^{*}\right) & \rightarrow \mathcal{M}(0,1) \\
{\left[\mathbb{D}^{*}, f, \Omega\right] } & \mapsto[\overline{\mathbb{C}}, \hat{f}]
\end{aligned}
$$

is a bijection.

Given $\mu \in L_{-1,1}^{\infty}\left(\mathbb{D}^{*}\right)$ as above, let $\hat{f}_{\mu}: \overline{\mathbb{C}} \rightarrow \overline{\mathbb{C}}$ be a quasiconformal map with Beltrami differential equal to $\mu$ in $\mathbb{D}^{*}$ and to 0 in $\mathbb{D}$. However, now we uniquely specify $\hat{f}_{\mu}$ with the normalizations $\hat{f}_{\mu}(0)=0, \hat{f}_{\mu}^{\prime}(0)=1$ and $\hat{f}_{\mu}(\infty)=\infty$. Denote

$$
f_{\mu}=\left.\hat{f}_{\mu}\right|_{\mathbb{D}}
$$

which is a conformal map; this map $f_{\mu}$ is independent of the choice of representative in the Teichmüller equivalence class. It can be shown that two Beltrami differentials $\mu$ and $\nu$ on $\mathbb{D}^{*}$ represent the same point of $T\left(\mathbb{D}^{*}\right)$ if and only if $f_{\mu}=f_{\nu}$ [31. Thus, $T\left(\mathbb{D}^{*}\right)$ can be identified with the moduli space of Example 2.6 up to a change of normalization, by applying the uniformization theorem to identify $R$ with $\overline{\mathbb{C}}$.

Define the spaces of abelian differentials

$$
A_{1}^{\infty}(\mathbb{D})=\left\{\alpha(z) d z \text { holomorphic on } \mathbb{D}: \sup _{z \in \mathbb{D}}\left(1-|z|^{2}\right)|\alpha(z)|<\infty\right\}
$$

and quadratic differentials

$$
A_{2}^{\infty}(\mathbb{D})=\left\{Q(z) d z^{2} \text { holomorphic on } \mathbb{D}: \sup _{z \in \mathbb{D}}\left(1-|z|^{2}\right)^{2}|Q(z)|<\infty\right\} .
$$


Denote the Schwarzian derivative of a conformal map $f$ by

$$
\mathcal{S}(f)=\frac{f^{\prime \prime \prime}}{f^{\prime}}-\frac{3}{2}\left(\frac{f^{\prime \prime}}{f^{\prime}}\right)^{2} .
$$

The Bers embedding is defined by

$$
\begin{aligned}
\beta: T\left(\mathbb{D}^{*}\right) & \rightarrow A_{2}^{\infty}(\mathbb{D}) \\
{[\mu] } & \mapsto \mathcal{S}\left(f_{\mu}\right) d z^{2} .
\end{aligned}
$$

where we use the Beltrami differential model of $T\left(\mathbb{D}^{*}\right)$. That is, $\beta=\mathcal{S} \circ \Phi$. It is a classical theorem of Bers that $\beta\left(T\left(\mathbb{D}^{*}\right)\right)$ is an open subset of $A_{2}^{\infty}(\mathbb{D})$; in fact this is a homeomorphism with respect to the so-called Teichmüller metric. Thus $T\left(\mathbb{D}^{*}\right)$ can be given a complex structure from $A_{2}^{\infty}(\mathbb{D})$.

Next, we recall another model of the universal Teichmüller space $T\left(\mathbb{D}^{*}\right)$ in terms of quasisymmetries.

Definition 4.8. An orientation-preserving homeomorphism $h$ of $\mathbb{S}^{1}$ is called a quasisymmetric mapping, iff there is a constant $k>0$, such that for every $\alpha$ and every $\beta$ not equal to a multiple of $2 \pi$, the inequality

$$
\frac{1}{k} \leq\left|\frac{h\left(e^{i(\alpha+\beta)}\right)-h\left(e^{i \alpha}\right)}{h\left(e^{i \alpha}\right)-h\left(e^{i(\alpha-\beta)}\right)}\right| \leq k
$$

holds. Let $\mathrm{QS}\left(\mathbb{S}^{1}\right)$ denote the set of quasisymmetric maps from $\mathbb{S}^{1}$ to $\mathbb{S}^{1}$.

The boundary values of a quasiconformal map are in general quasisymmetries [31]. By a classical result due to Beurling and Ahlfors [31, any quasisymmetry $h$ has a quasiconformal extension to $\mathbb{D}^{*}$. Another quasiconformal extension $E(h)$ was given by A. Douady and C. Earle [34] with the property that $E(T \circ h \circ S)=T \circ E(h) \circ S$ for any disk automorphisms $T$ and $S$.

Two Beltrami differentials $\mu$ and $\nu$ are Teichmüller equivalent if and only if the quasiconformal solutions $f^{\mu}: \mathbb{D}^{*} \rightarrow \mathbb{D}^{*}$ and $f^{\nu}: \mathbb{D}^{*} \rightarrow \mathbb{D}^{*}$ to the Beltrami equation on $\mathbb{D}^{*}$ are equal on $\mathbb{S}^{1}$ up to post-composition by a Möbius transformation of $\mathbb{S}^{1}$. That is, if and only if there is a Möbius transformation $T: \mathbb{D}^{*} \rightarrow \mathbb{D}^{*}$ such that $\left.T \circ f^{\mu}\right|_{\mathbb{S}^{1}}=\left.f^{\nu}\right|_{\mathbb{S}^{1}}$. Thus we may identify

$$
T\left(\mathbb{D}^{*}\right) \cong \mathrm{QS}\left(\mathbb{S}^{1}\right) / \operatorname{Möb}\left(\mathbb{S}^{1}\right) .
$$

The identification is given by $\left.[\mu] \mapsto f^{\mu}\right|_{\mathbb{S}^{1}}$. The inverse is obtained by applying the AhlforsBeurling extension theorem.

It is an important result that $\mathrm{QS}\left(\mathbb{S}^{1}\right)$ and $\mathrm{QS}\left(\mathbb{S}^{1}\right) / \mathrm{Möb}\left(\mathbb{S}^{1}\right)$ are groups under composition. Although the universal Teichmüller space has a topological structure (determined for example by the Teichmüller metric), it is not a topological group, since while right composition is continuous, left composition is not.

A remarkable property of the universal Teichmüller space is that it contains the Teichmüller spaces of all surfaces whose universal cover is the disk. This fact is obtained through the representation of surfaces by quotients of the disk by Fuchsian groups. Teichmüller theory can also be viewed as the deformation theory of Fuchsian groups [26, 31, 34]. In this paper we take instead the equivalent view of Teichmüller theory as the space of deformations of complex structures. 
4.3. The Teichmüller/rigged moduli space correspondence. We saw in Examples 2.4 and 2.6 that the universal Teichmüller space is a comparison moduli space. In fact, by work of Radnell and Schippers [43], this holds (up to a $\mathbb{Z}^{n}$ action) for Teichmüller spaces of Riemann surfaces with more boundary curves and higher genus. More precisely, the quotient of the Teichmüller space of genus $g$ surfaces bordered by $n$ closed curves by a $\mathbb{Z}^{n}$ action is the rigged moduli space $\widetilde{\mathcal{M}}(g, n)$. We outline these results here.

The case of $T\left(\mathbb{D}^{*}\right)$ as the moduli space of maps into $\overline{\mathbb{C}}$ is special, since all Riemann surfaces which are homeomorphic to the sphere are biholomorphic to the sphere. We now deal with the case that $g$ is non-zero. Given a bordered Riemann surface $\Sigma^{B}$ of type $(g, n)$ for $g \geq 1$ and $n \geq 1$, represent it as a subset of a punctured Riemann surface $\Sigma^{P}$ of type $(g, n)$, in such a way that the boundary $\partial \Sigma^{B}$ consists of $n$ quasicircles each encircling one puncture. This can always be done using a sewing procedure [43]. Given an element $\left[\Sigma^{B}, f, \Sigma_{1}^{B}\right] \in T\left(\Sigma^{B}\right)$, let $\hat{f}: \Sigma^{P} \rightarrow \Sigma_{1}^{P}$ be a quasiconformal map such that $\hat{f}$ is conformal on $\Sigma^{P} \backslash \operatorname{cl} \Sigma^{B}$ and equal to $f$ on $\Sigma^{B}$. Now fix a collection of conformal maps $\tau_{i}: \mathbb{D} \rightarrow \Sigma^{P}$ onto each of the connected components of the complement $\Sigma^{P} \backslash \mathrm{cl} \Sigma^{B}$. We then have the map

$$
\begin{aligned}
\Phi: T\left(\Sigma^{B}\right) & \rightarrow \widetilde{\mathcal{M}}(g, n) \\
{\left[\Sigma^{B}, f, \Sigma_{1}^{B}\right] } & \mapsto\left[\Sigma_{1}^{P},\left(\hat{f} \circ \tau_{1}, \ldots, \hat{f} \circ \tau_{n}\right)\right] .
\end{aligned}
$$

The pure Teichmüller modular group $\operatorname{PMod}\left(\Sigma^{B}\right)$ is the set of equivalence classes of quasiconformal maps $\rho: \Sigma^{B} \rightarrow \Sigma^{B}$ such that $\rho\left(\partial_{i} \Sigma^{B}\right)=\partial_{i} \Sigma^{B}$ as a set. Two such maps $\rho_{1}, \rho_{2}$ are equivalent $\rho_{1} \sim \rho_{2}$ if and only if they are homotopic rel boundary. The modular group acts discontinuously via $[\rho]\left[\Sigma^{B}, f, \Sigma_{1}^{B}\right]=\left[\Sigma^{B}, f \circ \rho^{-1}, \Sigma_{1}^{B}\right]$, and for any $[\rho]$ the induced map of $T\left(\Sigma^{B}\right)$ is a biholomorphism. Let DB denote the subset of $\operatorname{PMod}\left(\Sigma^{B}\right)$ generated by quasiconformal maps which are the identity on every boundary curves and are homotopic to the identity map. This group can be pictured as the set of elements twisting each boundary curve an integer number of times, and is isomorphic to $\mathbb{Z}^{n}$ except when $g=0$ and $n=1$ or $n=2$, in which case it is trivial or isomorphic to $\mathbb{Z}$ respectively.

We then have the following.

Theorem 4.9 ([43]). Let $\Sigma^{B}$ be a bordered Riemann surface. The map $\Phi: T\left(\Sigma^{B}\right) \rightarrow \widetilde{\mathcal{M}}(g, n)$ is a bijection up to a discrete group action. That is, if $\Phi\left(\left[\Sigma^{B}, f_{1}, \Sigma_{1}^{B}\right]=\Phi\left(\left[\Sigma^{B}, f_{2}, \Sigma_{2}^{B}\right]\right)\right.$ then there is $a[\rho] \in D B$ such that $[\rho]\left[\Sigma^{B}, f_{1}, \Sigma_{1}^{B}\right]=\left[\Sigma^{B}, f_{2}, \Sigma_{2}^{B}\right]$.

Here is a specific example.

Example 4.1 ([46]). Let $A$ be an annulus of finite modulus. The Teichmüller space $T(A) / \mathbb{Z}$ can be identified with the rigged moduli space $\widetilde{\mathcal{M}}(0,2)$.

Interestingly this has a semigroup structure (it is known as the Neretin-Segal semigroup) and every element can be decomposed as the product of a quasisymmetry of $\mathbb{S}^{1}$ and a bounded univalent function.

This example suggests the following problems, closely related to Problem 4.1;

Problem 4.3. Lift the conformally invariant functionals (3.1) to $T(A)$.

Of course this problem extends to Teichmüller space.

Problem 4.4. Extend the functionals (3.1) to Teichmüller spaces of bordered Riemann surfaces of type $(g, n)$. 
And again we can ask, similarly to Problem 4.2:

Problem 4.5. Does the Teichmüller space of bordered surfaces of type $(g, n)$ have a boundary, which corresponds under $\Phi$ to those riggings which map onto analytic arcs of quadratic differentials?

Problem 4.6. Can Theorem 4.9 be extended to Teichmüller spaces of more general surfaces, say those with infinite genus and/or infinitely many riggings? What conditions are necessary to make this work?

It is likely that a bound on the lengths of closed geodesics is a necessary condition; see Section 5.3 and Problem 5.3 ahead.

4.4. Some applications of the Teichmüller space/rigged moduli space correspondence. This identification of the moduli spaces appearing in conformal field theory (CFT) and in Teichmüller theory has far-reaching consequences. In particular, one can endow the rigged moduli space with a complex structure and show that the operation of sewing bordered Riemann surfaces together with quasisymmetries is holomorphic [43]. These are required for the construction of two-dimensional conformal field theory from vertex operator algebras. A comprehensive review can be found in [52]. The sewing technique is also of independent interest in Teichmüller theory.

On the other hand, the correspondence can be applied to transfer structures in CFT to Teichmüller theory. For example, one can obtain a fibre structure on Teichmüller space as follows. Let $\Sigma^{B}$ be a fixed Riemann surface. Define the map

$$
\begin{aligned}
\mathcal{C}: T\left(\Sigma^{B}\right) & \rightarrow T\left(\Sigma^{P}\right) \\
{\left[\Sigma^{B}, f, \Sigma_{1}^{B}\right] } & \mapsto\left[\Sigma^{P}, \hat{f}, \Sigma_{1}^{P}\right]
\end{aligned}
$$

where $\Sigma^{P}, \hat{f}$ and $\Sigma_{1}^{P}$ are determined from $\left[\Sigma^{B}, f, \Sigma_{1}^{B}\right]$ as above. Radnell and Schippers showed the following.

Theorem 4.10 ([45]). Let $\Sigma^{B}$ be a bordered surface of type $(g, n)$, and $\Sigma^{P}$ be a punctured surface of type $(g, n)$. Assume that $\Sigma^{B} \subset \Sigma^{P}$ and $\partial \Sigma^{B}$ consists of $n$ quasicircles each enclosing a distinct puncture in $\Sigma^{P}$.

(1) $\mathcal{C}$ is a holomorphic map with local holomorphic sections. The fibres $\mathcal{C}^{-1}(p)$ for $p \in$ $T\left(\Sigma^{P}\right)$ are complex submanifolds of $T\left(\Sigma^{B}\right)$.

(2) Let $p \in T\left(\Sigma^{P}\right)$. If $\left(\Sigma^{P}, g, \Sigma_{1}^{P}\right)$ is a representative of $p$, then the quotient $\mathcal{C}^{-1}(p) / D B$ is canonically bijective with $\mathcal{O}^{\mathrm{qc}}\left(\Sigma_{1}^{P}\right)$.

(3) The bijection in (2) is a biholomorphism.

In particular, this can be used to give holomorphic coordinates on $T\left(\Sigma^{B}\right)$. The proof uses a variational technique of F. Gardiner [15], which was based on an idea of Schiffer. The use of Gardiner-Schiffer variation to address holomorphicity issues in the rigged moduli space originates with Radnell [42].

Problem 4.7. Are there generalizations of Theorem 4.10 for Teichmüller spaces to more general Riemann surfaces, such as those with infinite genus and/or infinite number of boundary curves? 


\section{Weil-Petersson class Teichmüller space}

5.1. The Weil-Petersson class Teichmüller space. There exist many refinements of Teichmüller space, including for example the asymptotically conformal Teichmüller space [13, BMO-Teichmüller space [10, and $L^{p}$ Teichmüller spaces (references below). The $L^{2}$ case is usually referred to as the Weil-Petersson class Teichmüller space, which we motivate in this section. From now on we abbreviate this as WP-class.

The Weil-Petersson metric is a metric in the sense of Riemannian/Hermitian geometry, that is, an inner product at every tangent space. It is based on a representation of the tangent space to Teichmüller space by a set of quadratic differentials, which we must now describe. Fix a Riemann surface $\Sigma$. Given a holomorphic curve $t \mapsto \mu_{t}$ in the set of Beltrami differentials $L_{-1,1}^{\infty}(\Sigma)_{1}$, the derivative $\frac{d}{d t} \mu$ is in $L_{-1,1}^{\infty}(\Sigma)$. However, there is an enormous amount of redundancy in this model, since the difference between any pair of these might be tangent to the Teichmüller equivalence relation, and hence represent the same direction in Teichmüller space.

The following decomposition remedies the problem. Let $\lambda^{2}$ denote the hyperbolic metric on $\Sigma$. Define the harmonic Beltrami differentials by

$$
\Omega_{-1,1}(\Sigma)=\left\{\mu \in L_{-1,1}^{\infty}(\Sigma): \mu=\alpha / \lambda^{2}, \quad \alpha \text { a quadratic differential }\right\} .
$$

It can easily be verified that a quotient of a quadratic differential by the hyperbolic metric is a Beltrami differential, by writing them in local coordinates and verifying that the quotient satisfies the correct transformation rule. It is a classical result that we have the Banach space decomposition

$$
L_{-1,1}^{\infty}(\Sigma)_{1}=\Omega_{-1,1}(\Sigma) \oplus \mathcal{N}
$$

where $\mathcal{N}$ is the set of Beltrami differentials which are tangent to the Teichmüller equivalence relation. These are the so-called "infinitesimally trivial Beltrami differentials", which can be characterized precisely [31, 34].

Thus, the tangent space to $T(\Sigma)$ at $[\Sigma$, Id, $\Sigma]$ can be identified with $\Omega_{-1,1}(\Sigma)$. Furthermore, there is an open subset $U \subset \Omega_{-1,1}(\Sigma)$ containing 0 such that

$$
\begin{aligned}
\Psi: U & \rightarrow T(\Sigma) \\
\mu & \mapsto\left[\Sigma, f, \Sigma_{1}\right], \quad \text { where } \bar{\partial} f / \partial f=\mu
\end{aligned}
$$

is a biholomorphism from an open neighbourhood of 0 to an open neighbourhood of 0 . Applying right compositions by quasiconformal maps, one obtains a system of coordinates on $T(\Sigma)$ which is compatible with that obtained from the Bers embedding $\beta$. This is closely related to the so-called Ahlfors-Weill reflection. The tangent spaces at other points can be obtained by right composing by quasiconformal maps. These constructions can also all be lifted to the universal cover and expressed in terms of differentials invariant under Fuchsian groups.

For compact surfaces or compact surfaces with punctures, the Weil-Petersson metric is defined on the tangent space at the identity by the $L^{2}$ pairing of Beltrami differentials

$$
\langle\mu, \nu\rangle=\iint_{\Sigma} \bar{\mu} \nu d A_{\text {hyp }}, \quad \mu, \nu \in \Omega_{-1,1}(\Sigma)
$$

where $d A_{\text {hyp }}$ is the hyperbolic area measure [3]. The integral (5.3) is finite, because on compact surfaces $L^{\infty}$ Beltrami differentials are also $L^{2}$. This Hermitian inner product can be 
transferred to tangent spaces at other points in the Teichmüller space by right composition. In the compact/compact with punctures case, the Weil-Petersson metric has been much studied [78]. Ahlfors showed that the WP-metric is Kähler [3] and later computed its Ricci and scalar curvatures of holomorphic sections, and showed they are negative [4].

The Weil-Petersson inner product had not been defined on any other Teichmüller space until the turn of the millenium. The obstacle was that if the Riemann surface is not compact or compact with punctures, then the $L^{2}$ pairing need not be finite. Thus in order to define a Weil-Petersson metric one must restrict to a smaller Teichmüller space, so that tangent directions generate only $L^{2}$ Beltrami differentials. Nag and Verjovsky [37] showed that if one restricts to the subset of the universal Teichmüller space corresponding to diffeomorphisms of $\mathbb{S}^{1}$, then the WP metric converges on tangent directions. Equivalently, the corresponding representative quasisymmetries are smooth. However, this is only a heuristic principle, and the smoothness assumption is rather artificial from the point of view of Teichmüller theory. The correct analytic condition giving the largest universal Teichmüller space on which the WP metric converges is the subject of the next section.

5.2. Weil-Petersson class universal Teichmüller space. The investigation of $L^{2}$ Teichmüller spaces is due independently to G. Cui [9] and L. Takhtajan and L.-P. Teo [72]. H. Guo [22] and S.-A. Tang [75] extended some of the results to $L^{p}$ Teichmüller spaces.

The definitions of the WP-class Teichmüller space given by Takhtajan/Teo and Cui are rather different, but equivalent. Takhtajan/Teo give the definitions and complex structure in terms of $L^{2}$ harmonic Beltrami differentials, while Cui's definition is in terms of an $L^{2}$ condition on the quadratic differentials in the image of the Bers embedding.

Remark 5.1. In fact Takhtajan and Teo's approach defines a foliation of $T\left(\mathbb{D}^{*}\right)$ by right translates of the WP-class Teichmüller space.

Recall the space of differentials

$$
A_{1}^{2}(\mathbb{D})=\left\{\alpha(z) d z \text { holomorphic on } \mathbb{D}: \iint_{\mathbb{D}}|\alpha(z)|^{2}<\infty\right\}
$$

and quadratic differentials

$$
A_{2}^{2}(\mathbb{D})=\left\{Q(z) d z^{2} \text { holomorphic on } \mathbb{D}: \iint_{\mathbb{D}}\left(1-|z|^{2}\right)^{2}|Q(z)|^{2}<\infty\right\} .
$$

The coefficients of the differentials in $A_{1}^{2}(\mathbb{D})$ are in the Bergman space, and thus we use the same notation as in Section 3.3 (see Remark 3.9).

We begin with Cui's definition.

Definition 5.2. The WP-class universal Teichmüller space is defined to be

$$
T_{\mathrm{WP}}\left(\mathbb{D}^{*}\right)=\left\{[\mu] \in T\left(\mathbb{D}^{*}\right): \beta([\mu]) \in A_{2}^{2}(\mathbb{D})\right\} .
$$

Guo, Cui and Takhtajan/Teo also showed that $[\mu] \in T_{\mathrm{WP}}\left(\mathbb{D}^{*}\right)$ if and only if $f_{\mu}^{\prime \prime} / f_{\mu}^{\prime} \in A_{1}^{2}(\mathbb{D})$. Thus we have that

$$
[\mu] \in T_{\mathrm{WP}}\left(\mathbb{D}^{*}\right) \Leftrightarrow f_{\mu} \in \mathcal{O}_{\mathrm{WP}}^{\mathrm{qc}}(\mathbb{D}) .
$$

Although Takhtajan and Teo used a different definition, they also showed the equivalence of their definition with Definition [5.2, so we attribute the theorems below jointly.

Takhtajan/Teo and Cui independently showed that 
Theorem $5.3([9,72]) . A_{2}^{2}(\mathbb{D}) \subset A_{2}^{\infty}(\mathbb{D})$ and the inclusion is holomorphic.

Combined with the following, we get an analogue of the Bers embedding for $T_{\mathrm{WP}}\left(\mathbb{D}^{*}\right)$.

Theorem $5.4([9,72]) \cdot \beta\left(T_{\mathrm{WP}}\left(\mathbb{D}^{*}\right)\right)=\beta\left(T\left(\mathbb{D}^{*}\right)\right) \cap A_{2}^{2}(\mathbb{D})$. In particular, $\beta\left(T_{\mathrm{WP}}\left(\mathbb{D}^{*}\right)\right)$ is open.

Thus we have that $T_{\mathrm{WP}}\left(\mathbb{D}^{*}\right)$ has a complex structure inherited from $A_{2}^{2}(\mathbb{D})$.

Guo and Cui showed that the fact that $\beta([\mu]) \in A_{2}^{2}(\mathbb{D})$ is equivalent to the existence of a representative Beltrami differential which is $L^{2}$ with respect to the hyperbolic metric. Define for any Riemann surface $\Sigma$ with a hyperbolic metric $\lambda^{2}$

$$
L_{\text {hyp }}^{2}(\Sigma)=\left\{\mu \in L_{-1,1}^{\infty}(\Sigma): \iint_{\Sigma}|\mu|^{2} d A_{\text {hyp }}<\infty\right\}
$$

where $d A_{\text {hyp }}$ denotes hyperbolic area measure. In particular

$$
L_{\text {hyp }}^{2}\left(\mathbb{D}^{*}\right)=\left\{\mu \in L_{-1,1}^{\infty}\left(\mathbb{D}^{*}\right): \iint_{\mathbb{D}^{*}} \frac{|\mu|^{2}}{\left(1-|z|^{2}\right)^{2}} d A<\infty\right\} .
$$

We then have the following.

Theorem $5.5([9,72]) .[\mu] \in T_{\mathrm{WP}}\left(\mathbb{D}^{*}\right)$ if and only if $[\mu]$ has a representative $\mu \in L_{\mathrm{hyp}}^{2}\left(\mathbb{D}^{*}\right)$.

In fact, Guo and Cui gave stronger results in two directions. Guo showed such a result for $L^{p}$ differentials with respect to the hyperbolic metric, $p \geq 1$. Cui showed that the DouadyEarle extension of the boundary values of any representative conformal map $f_{\mu}$ has this property, and in fact satisfies a stronger integral estimate. Tang showed that the DouadyEarle extension is in $L^{p}$ and that the Bers embedding is holomorphic with respect to the intersection norm on $L^{p} \cap L^{\infty}$.

If $f_{\mu} \in \mathcal{O}^{\text {qc }}$, then the image of $\mathbb{S}^{1}$ under the unique homeomorphic extension of $f_{\mu}$ to $\mathrm{cl} D$ is a quasicircle. Although there are an astonishing number of non-trivially equivalent characterizations of quasicircles [18], there is no known geometric characterization of WeilPetersson class quasicircles.

Problem 5.1. Characterize quasicircles of the form $f_{\mu}\left(\mathbb{S}^{1}\right)$ for $[\mu] \in T_{\mathrm{WP}}(\mathbb{D})$ (equivalently, for $\left.f_{\mu} \in \mathcal{O}_{\mathrm{WP}}^{\mathrm{qc}}\right)$ via analytic or geometric conditions on the set itself.

Radnell, Schippers, and Staubach [49] showed that a Weil-Petersson class quasicircle is a rectifiable chord-arc curve, but this is unlikely to be sufficient.

We also define the WP-class quasisymmetries as follows.

Definition 5.6. We say that $\phi \in \mathrm{QS}\left(\mathbb{S}^{1}\right)$ is Weil-Petersson class if its corresponding Teichmüller space representative is in $T_{\mathrm{WP}}\left(\mathbb{D}^{*}\right)$. Denote the set of WP-class quasisymmetries by $\mathrm{QS}_{\mathrm{WP}}\left(\mathbb{S}^{1}\right)$.

Cui and Takhtajan/Teo showed that, like quasisymmetries, these are closed under composition and inverse.

Theorem $5.7([9,72])$. $\mathrm{QS}_{\mathrm{WP}}\left(\mathbb{S}^{1}\right)$ is a group.

A stronger result was obtained by Takhtajan and Teo:

Theorem $5.8([72])$. $\mathrm{QS}_{\mathrm{WP}}\left(\mathbb{S}^{1}\right) / M \ddot{o} b\left(\mathbb{S}^{1}\right)$ is a topological group. 
This is in contrast to $\mathrm{QS}\left(\mathbb{S}^{1}\right) / \mathrm{Möb}\left(\mathbb{S}^{1}\right)$, which is not a topological group.

Remark 5.9. Takhtajan and Teo also showed that $\mathrm{QS}_{\mathrm{WP}}\left(\mathbb{S}^{1}\right) / \mathbb{S}^{1}$ is a topological group, and it can be identified naturally with the WP-class universal Teichmüller curve.

The WP-class quasisymmetries were characterized by Y. Shen as follows, answering a problem posed by Takhtajan and Teo.

Theorem 5.10 ([71]). Let $\phi: \mathbb{S}^{1} \rightarrow \mathbb{S}^{1}$ be a homeomorphism. $\phi \in \mathrm{QS}_{\mathrm{WP}}\left(\mathbb{S}^{1}\right)$ if and only if $\phi$ is absolutely continuous and $\log \phi^{\prime}$ is in the Sobolev space $H^{1 / 2}\left(\mathbb{S}^{1}\right)$.

Further characterizations (e.g. in terms of the composition operator associated with $\phi$ ) were given by $\mathrm{Y}$. Hu and Y. Shen [24].

It was also shown that right composition is biholomorphic.

Theorem $5.11([9,72])$. Right composition $\left(\bmod M \ddot{o} b\left(\mathbb{S}^{1}\right)\right)$ in $T\left(\mathbb{D}^{*}\right)$ by a fixed element $h \in \mathrm{QS}_{\mathrm{WP}}\left(\mathbb{D}^{*}\right)$ is a biholomorphism.

This theorem combined with Theorem 5.4 was used by Cui to define the Weil-Petersson pairing on any tangent space, by using the pairing in $A_{2}^{2}(\mathbb{D})$ at the identity, and then applying the above theorem to define a right-invariant metric.

Takhtajan and Teo's approach, on the other hand, defined the complex structure in terms of local charts into the space of harmonic Beltrami differentials, which are defined by

$$
H_{-1,1}\left(\mathbb{D}^{*}\right)=\left\{\mu=\left(1-|z|^{2}\right)^{2} \overline{Q(z)}: \mu \in L_{\text {hyp }}^{2}\left(\mathbb{D}^{*}\right)\right\} .
$$

This is non-trivial as it must be shown that the transition functions of the charts are biholomorphisms. As in the classical case, their proof relies on the use of the Ahlfors-Weill reflection.

Their construction also gives a description of the tangent space in terms of harmonic Beltrami differentials. By Theorem 5.3 we immediately obtain

Theorem $5.12([72])$.

$$
L_{-1,1}^{\infty}\left(\mathbb{D}^{*}\right) \cap L_{\text {hyp }}^{2}\left(\mathbb{D}^{*}\right)=H_{-1,1}\left(\mathbb{D}^{*}\right) \oplus\left(\mathcal{N} \cap L_{\text {hyp }}^{2}\left(\mathbb{D}^{*}\right)\right) .
$$

This also shows that the tangent space at $[0]$ to $T_{\mathrm{WP}}\left(\mathbb{D}^{*}\right)$ can be identified with $H_{-1,1}\left(\mathbb{D}^{*}\right)$. This has finite Weil-Petersson pairing (5.3) by definition. By applying the holomorphic right translation, Takhtajan and Teo obtain a right invariant inner product at all points in $T_{\mathrm{WP}}\left(\mathbb{D}^{*}\right)$.

In fact, they extended this complex structure and Hermitian metric to all of $T\left(\mathbb{D}^{*}\right)$ in the following way. A neighbourhood of $[0]$ in $T\left(\mathbb{D}^{*}\right)$ can be obtained under the map $\Psi$ (5.2), with $\Omega_{-1,1}\left(\mathbb{D}^{*}\right)$ replaced by $H_{-1,1}\left(\mathbb{D}^{*}\right)$. Using right composition, the charts patch together to give a complex structure compatible with the Bers embedding. However, the topology and complex structure are not equivalent to the standard one on $T\left(\mathbb{D}^{*}\right)$. Indeed, $T\left(\mathbb{D}^{*}\right)$ consists of uncountably many disjoint translates of $T_{\mathrm{WP}}\left(\mathbb{D}^{*}\right)$, each of which is a connected component of $T\left(\mathbb{D}^{*}\right)$ with this new topology. In their definition, $T_{\mathrm{WP}}\left(\mathbb{D}^{*}\right)$ appears as the connected component of the identity. This accounts for the convergence of the WP pairing, since at any given point there are far fewer tangent vectors than there are when $T\left(\mathbb{D}^{*}\right)$ is given the standard complex structure. That is, the directions on which the WP pairing diverges are excluded. 
It must be emphasized that these constructions require a great deal of analysis; one cannot simply make small adjustments to classical theorems of Teichmüller theory.

Finally, Takhtajan and Teo showed (improving on Nag and Verjovsky's result, which held only in the diffeomorphism case)

Theorem $5.13\left([37,[72])\right.$. The Weil-Petersson metric on $T\left(\mathbb{D}^{*}\right)$ (with the new complex structure and topology) is Kähler. In particular the Weil-Petersson metric on $T_{\mathrm{WP}}\left(\mathbb{D}^{*}\right)$ is Kähler.

In fact they gave different explicit formulas for the Kähler potential, and computed the Ricci curvatures. We will return to this in Section 5.4.

5.3. Higher genus Weil-Petersson class Teichmüller spaces. It is possible to extend the Weil-Petersson metric to a much wider class of surfaces, again by obtaining an $L^{2}$ theory. Radnell, Schippers and Staubach [47, 48, 50, 51] did this for bordered surfaces of type $(g, n)$. M. Yanagishita [79] extended the $L^{p}$ theory of Guo [22] and Tang [75] to surfaces satisfying "Lehner's condition", which includes bordered surfaces of type $(g, n)$. In the $L^{2}$-setting the two Teichmüller spaces are the same as sets, but the constructions of the complex structures are rather different. Yanagishita constructs the complex structure from $L^{p}$ quadratic differentials under the image of the Bers embedding, following the approach of Cui, Guo and Tang. Radnell, Schippers, and Staubach constructed the complex structure in two equivalent ways: using harmonic Beltrami differentials (along the lines of Takhtajan/Teo), and by refining the fiber structure of Theorem 4.10. As in the classical $L^{\infty}$ case, the complex structures arising from the Bers embedding into quadratic differentials and from harmonic Beltrami differentials should be equivalent, but this has not yet been established.1.

The Weil-Petersson class Teichmüller space is specified by boundary behaviour. To describe this we need a local coordinate near the boundary.

Definition 5.14. Let $\Sigma$ be a bordered surface of type $(g, n)$. A collar neighbourhood of a boundary curve $\partial_{i} \Sigma$ is a doubly connected open set in $\Sigma$, one of whose boundaries is $\partial_{i} \Sigma$ and the other is an analytic curve in the interior of $\Sigma$. A collar chart of a bordered surface $\Sigma$ is a conformal map $\zeta: U \rightarrow\{1<|z|<r\}$ for some $r>1$ which extends continuously to $\partial_{i} \Sigma$.

It is possible to show that the chart extends to a conformal map of an open neighbourhood of $\mathbb{S}^{1}$ into the double of $\Sigma$.

We define the WP-class Teichmüller space in two steps.

Definition 5.15. Let $\Sigma$ and $\Sigma_{1}$ be bordered surfaces of type $(g, n)$. A quasiconformal map $f: \Sigma \rightarrow \Sigma_{1}$ is called refined if for each pair of boundary curves $\partial_{i} \Sigma, \partial_{j} \Sigma_{1}$ such that $f\left(\partial_{i} \Sigma\right)=$ $\partial_{j} \Sigma_{1}$, there are collar charts $\zeta_{i}, \eta_{j}$ of $\partial_{i} \Sigma$ and $\partial_{j} \Sigma_{1}$ respectively such that $\left.\eta_{j} \circ f \circ \zeta_{i}^{-1}\right|_{\mathbb{S}^{1}} \in$ $\mathrm{QS}_{\mathrm{WP}}\left(\mathbb{S}^{1}\right)$. Denote the set of such quasiconformal maps by $\mathrm{QC}_{r}(\Sigma)$.

If the condition holds for one collar chart at a boundary $\partial_{i} \Sigma$, then it holds for all of them.

Definition 5.16. Let $\Sigma$ be a bordered surface of type $(g, n)$. The WP-class Teichmüller space of $\Sigma$ is

$$
T(\Sigma)=\left\{\left(\Sigma, f, \Sigma_{1}\right): f \in \mathrm{QC}_{r}(\Sigma)\right\} / \sim
$$

where $\sim$ is the usual Teichmüller equivalence.

\footnotetext{
${ }^{1}$ See Footnote 3
} 
A different definition was given by Yanagishita [79], for $L^{p}$ spaces for $p \geq 1$. It was phrased in terms of Fuchsian groups satisfying a condition he terms "Lehner's condition". We will restate Yanagishita's approach in its equivalent form on the Riemann surface, for consistency of presentation. Let $\Sigma^{*}$ denote the double of the Riemann surface $\Sigma$ (if $\Gamma$ is the Fuchsian group such that $\Sigma=\mathbb{D}^{*} / \Gamma$, then $\left.\Sigma^{*}=\mathbb{D} / \Gamma\right)$.

Definition 5.17 (Lehner's condition). A Riemann surface $\Sigma$ covered by the disk $\mathbb{D}^{*}$ satisfies Lehner's condition if the infimum of the hyperbolic lengths of the simple closed geodesics is strictly greater than 0 .

Definition 5.18. Let $\Sigma$ be a Riemann surface covered by the disk satisfying Lehner's condition. The p-integrable Teichmüller space $T^{p}(\Sigma)$ is the subset of $T(\Sigma)$ consisting of elements $\left[\Sigma, f, \Sigma_{1}\right]$ such that there is a representative $\left(\Sigma, f, \Sigma_{1}\right)$ such that the Beltrami differential of $f$ is in $L^{p}$ with respect to the hyperbolic metric.

Yanagishita also showed that the $L^{p}$ representative is given by the Douady-Earle extension of the boundary values of the lift to $\mathbb{D}^{*}$. We will see shortly that it agrees with the definition above in the special case of $p=2$ and bordered surfaces of type $(g, n)$.

Radnell, Schippers and Staubach obtained the following analogue of Theorem 5.5, using sewing techniques and the lambda lemma.

Theorem 5.19 ([50]). Let $\Sigma$ be a bordered Riemann surface of type $(g, n)$. Then $f \in$ $\mathrm{QC}_{r}(\Sigma)$ if and only if it is homotopic rel boundary to a quasiconformal map whose Beltrami differential is in $L_{\mathrm{hyp}}^{2}(\Sigma)$.

Since bordered surfaces of type $(g, n)$ satisfy Lehner's condition, by Theorem 5.19 , the Definitions 5.16 and 5.18 are equivalent for $p=2$ and bordered surfaces of this type.

The two approaches to the complex structure are rather different. Yanagishita's approach involves the following theorem. Let $\Sigma^{*}$ denote the double of $\Sigma$. Define $L_{\text {hyp }}^{p}(\Sigma)$ to be the set of Beltrami differentials on $\Sigma$ which are $L^{p}$ with respect to the hyperbolic area measure. Following [79] denote

$$
\operatorname{Ael}^{p}(\Sigma)=L_{-1,1}^{\infty}(\Sigma)_{1} \cap L_{\mathrm{hyp}}^{p}(\Sigma) .
$$

The intersection norm $\|\cdot\|_{p}+\|\cdot\|_{\infty}$ induces a topology on $T^{p}(\Sigma)$. Furthermore let

$$
A_{2}^{p}\left(\Sigma^{*}\right)=\left\{\alpha \text { a quadratic differential on } \Sigma^{*}: \iint_{\Sigma^{*}} \lambda^{-2}|\alpha|^{p}<\infty\right\} .
$$

(recall that $\lambda^{2}$ is the hyperbolic metric).

Theorem 5.20 ([79]). For $p \geq 2$, the restriction of the Bers embedding $\beta$ to $T^{p}(\Sigma)$ is a homeomorphism onto its image in $A_{2}^{p}\left(\Sigma^{*}\right)$ with respect to the Ael ${ }^{p}$ norm.

This induces a complex structure on $T^{p}(\Sigma)$, and in particular on $T_{\mathrm{WP}}(\Sigma)$ for bordered Riemann surfaces of type $(g, n)$. Furthermore, Yanagishita showed that right composition is a biholomorphism with respect to this structure, for $p \geq 2$. Thus, although the tangent space structure is not treated in the paper [79], it is possible to define a WP-pairing on the tangent space at $[0]$ using the $\Gamma$-invariant subspace of $A_{2}^{2}(\mathbb{D})$ as a model, and then using right composition to obtain a right-invariant metric at every point. 
Radnell, Schippers, and Staubach gave two other complex structures for bordered surfaces of type $(g, n)$, which are equivalent to each other. In [47, 48], it was shown that the fiber structure of Theorem 4.10 passes down to $T_{\mathrm{WP}}(\Sigma)$ for bordered Riemann surfaces of type $(g, n)$. That is, one may view $T_{\mathrm{WP}}(\Sigma)$ as fibred over $T\left(\Sigma^{P}\right)$ for a compact surface with punctures $\Sigma^{P}$, such that the fibres $\mathcal{C}^{-1}(p)$ modulo a discrete group action are biholomorphic to $\mathcal{O}_{\mathrm{WP}}^{\mathrm{qc}}\left(\Sigma_{1}^{P}\right)$. This can be used to construct a Hausdorff, second countable topology on $T_{\mathrm{WP}}(\Sigma)$, and a complex Hilbert manifold structure [47]. The advantage of this approach is that it is very flexible and constructive, and explicit coordinates can be given in terms of Gardiner-Schiffer variation.

In [51], Radnell, Schippers, and Staubach showed that this fiber structure is compatible with that obtained by harmonic Beltrami differentials and right translation, analogous to both the classical case and Takhtajan and Teo's approach on $T_{\mathrm{WP}}\left(\mathbb{D}^{*}\right)$. We briefly describe this below, as well as the description of the tangent spaces.

In all of the following theorems, $\Sigma$ is a bordered surface of type $(g, n)$.

Theorem 5.21 ([51]). $H_{-1,1}(\Sigma) \subseteq \Omega_{-1,1}(\Sigma)$ and inclusion is holomorphic. Furthermore

$$
L_{-1,1}^{\infty}(\Sigma) \cap L_{\text {hyp }}^{2}(\Sigma)=H_{-1,1}(\Sigma) \oplus\left(\mathcal{N} \cap L_{\text {hyp }}^{2}(\Sigma)\right) .
$$

Theorem 5.22 ([51]). Let $\alpha_{t}$ be any holomorphic curve in $T_{\mathrm{WP}}(\Sigma)$ for $t$ in a disk centered at 0 , such that $\alpha_{0}=[0]$. There is an open disk $D$ centered at zero so that for each $t \in D$, $\alpha_{t}$ has a representative which is in $L_{\text {hyp }}^{2}(\Sigma) \cap L_{-1,1}^{\infty}(\Sigma)$, which is a holomorphic curve in the Hilbert space $L_{\text {hyp }}^{2}(\Sigma)$ and the Banach space $L_{-1,1}^{\infty}(\Sigma)$.

Thus the tangent space at the identity is described by $H_{-1,1}(\Sigma)$. This can be right translated, and also leads to a complex structure as in the the classical case.

The harmonic Beltrami differentials also induce local coordinates. For $\mu \in H_{-1,1}(\Sigma)$ let $f_{\mu}: \Sigma \rightarrow \Sigma_{1}$ denote a quasiconformal solution to the Beltrami equation.

Theorem 5.23 ([51]). There is an open neighbourhood of $U$ of 0 in $H_{-1,1}(\Sigma)$ such that the map $\mu \mapsto\left[\Sigma, f_{\mu}, \Sigma_{1}\right] \in T_{\mathrm{WP}}(\Sigma)$ obtained by solving the Beltrami equation is a biholomorphism onto its image. Furthermore, change of base point is a biholomorphism. This describes a system of complex coordinates which endows $T_{\mathrm{WP}}(\Sigma)$ with a complex Hilbert manifold structure. This complex structure is compatible with the complex structure obtained from the fiber structure.

Corollary $5.24([51]) . T_{\mathrm{WP}}(\Sigma)$ has a finite Weil-Petersson pairing on each tangent space.

Remark 5.25. In particular, this shows that if one had defined the complex structure using harmonic Beltrami differentials in the first place, then $T_{\mathrm{WP}}(\Sigma)$ would have a holomorphic fiber structure with fibers $(\bmod \mathrm{DB})$ biholomorphic to $\mathcal{O}_{\mathrm{WP}}^{\mathrm{qc}}\left(\Sigma_{1}^{P}\right)$ (and hence locally biholomorphic to $\left.\left(\mathcal{O}_{\mathrm{WP}}^{\mathrm{qc}}\right)^{n}\right)$. The analogous result for the classical $L^{\infty}$ case is Theorem 4.10 above; even in the $L^{\infty}$ case it is non-trivial.

The above discussion leads naturally to the following problems.

Problem 5.2. Show that the complex structure on $T_{\mathrm{WP}}(\Sigma)$ induced by harmonic Beltrami differentials $H_{-1,1}(\Sigma)$ and right composition is equivalent to that induced by the Bers embedding into hyperbolically $L^{2}$ quadratic differentials. 2 
Problem 5.3. Can the holomorphic fiber structure of Theorem 4.10 be extended to more general surfaces satisfying Lehner's condition, in the WP-class $/ L^{p}$ case? For example, to surfaces of infinite genus and/or infinitely many boundary curves?

Since Takhtajan and Teo obtain a foliation of $T\left(\mathbb{D}^{*}\right)$ by translates of $T_{\mathrm{WP}}\left(\mathbb{D}^{*}\right)$ we are led to ask:

Problem 5.4. Can $T(\Sigma)$ be endowed with a complex Hilbert manifold structure, such that $T_{\mathrm{WP}}(\Sigma)$ is the connected component of the identity, and the other connected components are right translates of $T_{\mathrm{WP}}(\Sigma)$ ?

5.4. Kähler potential of Weil-Petersson metric. In this section we give a brief overview of some geometric problems associated with the Weil-Petersson metric.

Ahlfors [3] showed that for compact Riemann surfaces the Weil-Petersson metric is Kähler. In the commentary to his collected works he stated that André Weil also had a proof but had not published it. Later Ahlfors computed the curvatures of holomorphic sections and the Ricci curvature [4].

Kirillov and Yuriev [29] sketched a generalization of the period mapping in Teichmüller theory to Diff( $\left(\mathbb{S}^{1}\right) / \operatorname{Möb}\left(\mathbb{S}^{1}\right)$. Some aspects were filled out and extended to the full universal Teichmüller space by S. Nag [35] and S. Nag and D. Sullivan in [36]. In this formulation, the period map is a map from Teichmüller space into an infinite-dimensional Siegel disk, which is a set of bounded, symmetric operators $Z$ such that $I-Z \bar{Z}$ is positive-definite, analogous to the period mapping for compact Riemann surfaces. Nag and Sullivan [36] indicated that this period map is holomorphic by proving Gâteaux holomorphicity. The first complete proof of holomorphicity of the Kirillov-Yuriev-Nag-Sullivan period mapping was given by Takhtajan and Teo [72], in both the Weil-Petersson and classical setting.

In 23] D. K. Hong and S. G. Rajeev showed that the Siegel disk possesses a natural Kähler metric, whose Kähler potential is given, up to a multiplicative constant, by $\log \operatorname{det}(I-Z \bar{Z})$. Kirillov and Yuriev [29] and Nag [35] showed that the pull-back of a natural Kähler metric on the infinite Siegel disk is the Weil-Petersson metric. Thus $\log \operatorname{det}(I-Z \bar{Z})$ is also a Kähler potential for the Weil-Petersson metric. Hong and Rajeev noted that Diff $\left(\mathbb{S}^{1}\right) / \operatorname{Möb}\left(\mathbb{S}^{1}\right)$ was not complete with respect to the Kähler metric, which indicated that it was not the correct analytic setting for the Weil-Petersson metric.

The completion of $\operatorname{Diff}\left(\mathbb{S}^{1}\right) / \operatorname{Möb}\left(\mathbb{S}^{1}\right)$ with respect to the Weil-Petersson metric is $T_{\mathrm{WP}}\left(\mathbb{D}^{*}\right)$, as was demonstrated in [72]. In [72] Takhtajan and Teo also proved the striking result that the period mapping $Z$ is in fact the Grunsky operator. In particular, this implies that a constant multiple of the Fredholm determinant (3.3) is a Kähler potential for the Weil-Petersson metric. Finally, Takhtajan and Teo [72] and Shen [70] independently showed that the Grunsky operator $Z$ is Hilbert-Schmidt if and only if the corresponding Teichmüller representative is WP-class. This is a very satisfying result because this is exactly the condition required in order for $Z \bar{Z}$ to be trace-class and hence that $\operatorname{det}(I-Z \bar{Z})$ exists.

This leads us to the following natural problems:

\footnotetext{
${ }^{2}$ See Footnote 3
} 
Problem 5.5. Is the Weil-Petersson metric on the $L^{2}$ Teichmüller space of bordered surfaces of type $(g, n)$ Kähler? More generally, is this true for surfaces satisfying Lehner's condition?

Problem 5.6. Compute the sectional or Ricci curvatures of the Weil-Petersson metric for bordered surfaces of type $(g, n)$, or more generally those satisfying Lehner's condition 3

These problems are closely related to the following.

Problem 5.7. Is there a generalization of the Kirillov-Yuriev-Nag-Sullivan period mapping to Teichmüller spaces of bordered surfaces of type $(g, n)$, or more general surfaces? Can one obtain an analogous Kähler potential from this period mapping?

Note that generalizations of the Grunsky matrix to higher genus surfaces have been obtained by K. Reimer and E. Schippers [54], and for genus zero surfaces with $n$ boundary curves by Radnell, Schippers and Staubach [53].

5.5. Applications of Weil-Petersson class Teichmüller theory. The Weil-Petersson Teichmüller space has recently attracted a great deal of attention, in part because of its intrinsic importance to Teichmüller theory, and in part because of its many applications. We sketch some of these now.

In potential theory, the Weil-Petersson class domains are precisely those on which the Fredholm determinant of function theory exists, and therefore on which it is a viable tool in potential theory. In Teichmüller theory, the Weil-Petersson metric has been an important tool in the investigation of the geometry of Teichmüller space, see e.g. S. Wolpert [78]. It is now available in vastly greater generality.

There are also various physical applications. It has been suggested by several authors that the universal Teichmüller space could serve as a basis for a non-perturbative formulation of bosonic string theory. See Hong and Rajeev [23], M. G. Bowick and S. G. Rajeev [7], and the (somewhat dated) review of O. Pekonen [39]; for a review of connections to conformal field theory see Markina and Vasil'ev [32]. The $g$-loop scattering amplitude in string theory can be expressed as an integral over Teichmüller space [23], and thus a non-perturbative formulation might be given on the universal Teichmüller space, since it contains all other Teichmüller spaces. Hong and Rajeev also propose that the computation of the scattering amplitudes should involve the exponential of the Kähler potential discussed in the previous section. As observed above, it was known already to Hong and Rajeev that Diff $\left(\mathbb{S}^{1}\right) / \mathrm{Möb}\left(\mathbb{S}^{1}\right)$ is not complete with respect to the Kähler metric (which we now know to be the Weil-Petersson metric). The correct analytic setting for Hong and Rajeev's proposal thus appears to be the Weil-Petersson class Teichmüller space.

\footnotetext{
${ }^{3}$ After this chapter was submitted in May 2016, the paper [80] of M. Yanagishita appeared, in which it was shown that for surfaces satisfying Lehner's condition, the Weil-Petersson metric is indeed Kähler and the sectional and Ricci curvatures are negative. The convergent Weil-Petersson metric was obtained independently of Radnell, Schippers and Staubach [50, 51. Yanagishita 80, also showed that the complex structure from harmonic Beltrami differentials is compatible with the complex structure from the Bers embedding. When combined with the results of [51, this apparently shows that these two complex structures are equivalent to that obtained from fibrations over the compact surfaces for surfaces of type $(g, n)$.
} 
The Weil-Petersson Teichmüller space also has deep connections with two-dimensional conformal field theory as formulated by Segal, Kontsevich, Vafa and others (see [25] for a review of the literature in the formulation of CFT). Work of Radnell, Schippers and Staubach has established that the Weil-Petersson class rigged moduli space is the completion of the analytically rigged moduli space of Friedan/Shenker/Segal/Vafa, and is also the largest space on which constructions in conformal field theory can be carried out. These include for example sewing properties of the determinant line bundle over the rigged moduli space and the existence of local holomorphic sections. A review of this work can be found in [52].

Finally, there are applications to fluid mechanics and infinite dimensional groups of diffeomorphisms. The setting for this is a deep insight of Arnol'd, namely that the geodesic equations on infinite-dimensional diffeomorphism groups are analogous to the Euler equations of fluid mechanics [28]. Different choices of groups and metrics lead to different geodesic equations, which in turn are different systems of partial differential equations [28, Table 4.1]. See E. Grong, I. Markina and A. Vasiliev [20] for a survey of choices on Diff( $\left(\mathbb{S}^{1}\right) / \operatorname{Möb}\left(\mathbb{S}^{1}\right)$ and their relation to sub-Riemannian geometry. In the case of the Weil-Petersson metric on $\operatorname{Diff}\left(\mathbb{S}^{1}\right) / \mathbb{S}^{1}$, the geodesic equations are related to the $\mathrm{KdV}$ equation. M. E. Schonbek, A. N. Todorov and J. P. Zubelli [69] were able to obtain long-term solutions to the KdV equation using this connection. F. Gay-Balmaz [16] was able to obtain global existence and uniqueness of the geodesics, and applied the Euler-Poincaré reduction process to obtain the spatial representation of the geodesics. Further important and interesting applications in this direction were given by A. Figalli [14], F. Gay-Balmaz and T. S. Ratiu [17], and S. Kushnarev [30].

We note that an important technical problem in this direction was solved by Shen [71]. Much of the analysis in the fluid mechanical models above has involved the assumption that the corresponding quasisymmetries were in $H^{3 / 2-\epsilon}\left(\mathbb{S}^{1}\right)$ for $\epsilon>0$, and it was an open question whether the quasisymmetries in the Weil-Petersson class Teichmüller space would be precisely those in $H^{3 / 2}\left(\mathbb{S}^{1}\right)$. Theorem 5.10 of Shen above gave the correct characterization, and in the same paper [71] he also showed that there are WP-class quasisymmetries which are not in $H^{3 / 2}\left(\mathbb{S}^{1}\right)$.

\section{Conclusion}

6.1. Concluding remarks. In this paper, we have given a number of examples of the general phenomenon of comparison moduli spaces in geometric function theory and moduli spaces of Riemann surfaces. We have seen that this concept spontaneously arises in both modern and classical complex analysis. We have also attempted to illustrate how this notion of moduli space captures many complex analytic phenomena in a simple way.

We would like to conclude with another observation. One is struck by the pervasive relevance of classical function theory. We have seen, for example, the unwitting re-invention in two-dimensional conformal field theory of the Teichmüller space of bordered surfaces and conformal welding, and the use of Schiffer's variational technique to construct a complex structure on Teichmüller space and the rigged moduli space of conformal field theory. We have also seen the Fredholm determinant of classical potential theory - as reformulated by Schiffer - emerge as a fundamental geometric object on moduli spaces of Riemann surfaces. These geometric problems in turn require the formulation and solution of analytic problems 
which can only be approached with function theory. Other examples spring readily to the mind of any mathematician with their ear to the ground.

We cannot express this in any better way than Ahlfors did [2]: "We start out from a purely classical problem, and we place it in a much more general modern setting, sometimes in a form that would not have been available to a classical mathematician. When the generalized problem is analyzed, it turns out to lead forcefully to a new and evidently significant problem in the original purely classical framework. In other words, we are faced with new evidence of the scope and fertility of classical analysis."

\section{REFERENCES}

[1] L. V. Ahlfors and L. Sario, Riemann surfaces, Princeton University Press, 1960.

[2] L. V. Ahlfors, Classical and contemporary analysis. SIAM Rev. 3 (1961) 1 - 9.

[3] L. V. Ahlfors, Some remarks on Teichmller's space of Riemann surfaces. Ann. of Math. (2) $\mathbf{7 4} 1961$ $171-191$.

[4] L. V. Ahlfors, Curvature properties of Teichmüller's space. J. Analyse Math. 9 1961/1962 $161-176$.

[5] Lars V. Ahlfors, Conformal invariants: topics in geometric function theory. McGraw-Hill Series in Higher Mathematics. McGraw-Hill Book Co., New York-Düsseldorf-Johannesburg, 1973.

[6] S. Bergman and M. Schiffer, Kernel functions and conformal mapping. Compositio Math. 8, (1951), $205-249$.

[7] M. J. Bowick and S. G. Rajeev, The holomorphic geometry of closed bosonic string theory and $\operatorname{Diff}\left(\mathbb{S}^{1}\right) / \mathbb{S}^{1}$. Nuclear Phys. B 293 (1987), no. 2, 348 - 384.

[8] R. Courant, Dirichlet's principle, conformal mapping, and minimal surfaces. With an appendix by M. Schiffer. Reprint of the 1950 original. Springer-Verlag, New York-Heidelberg, 1977.

[9] G. Cui, Integrably asymptotic affine homeomorphisms of the circle and Teichmüller spaces, Sci. China Ser. A 43 (2000), no. 3, 267-279.

[10] G. Cui and M. Zinsmeister, BMO-Teichmüller spaces. Illinois J. Math. 48 (2004), no. 4, 1223 - 1233.

[11] V. N. Dubinin, Condenser capacities and symmetrization in geometric function theory. Translated from the Russian by Nikolai G. Kruzhilin. Springer, Basel, 2014.

[12] P. Duren, Univalent functions, Grundlehren der Mathematischen Wissenschaften 259, Springer-Verlag, New York (1983).

[13] C. J. Earle, F. P. Gardiner, and N. Lakic, Asymptotic Teichmller space. I. The complex structure. In the tradition of Ahlfors and Bers (Stony Brook, NY, 1998), 17 - 38, Contemp. Math., 256, Amer. Math. Soc., Providence, RI, 2000.

[14] A. Figalli, On flows of $H^{\frac{3}{2}}$-vector fields on the circle. Math. Ann. 347 (2010), 43-57.

[15] F. P. Gardiner, Schiffer's interior variation and quasiconformal mapping, Duke Math. J. 42 (1975), $371-380$.

[16] F. Gay-Balmaz, Infinite dimensional geodesic flows and the universal Teichmller space. Thèse École polytechnique fédérale de Lausanne EPFL, nr. 4254 (2009). DOI:10.5075/epfl-thesis- 4254.

[17] F. Gay-Balmaz and T. S. Ratiu, The geometry of the universal Teichmüller space and the Euler-WeilPetersson equation, Adv. Math. 279 (2015), 717-778.

[18] F. W. Gehring and K. Hag, The ubiquitous quasidisk. With contributions by Ole Jacob Broch. Mathematical Surveys and Monographs, 184. American Mathematical Society, Providence, RI, 2012.

[19] A. Z. Grinshpan, Logarithmic geometry, exponentiation, and coefficient bounds in the theory of univalent functions and non-overlapping domains in Handbook of Complex Analysis: Geometric Function Theory, Vol I., edited by R. Kühnau, North-Holland, Amsterdam, 2002.

[20] E. Grong, I. Markina, and A. Vasil'ev, Sub-Riemannian structures corresponding to Kählerian metrics on the universal Teichmüller space and curve. "60 years of analytic functions in Lublin" in memory of our professors and friends Jan G. Krzyż, Zdzisław Lewandowski and Wojciech Szapiel, 97116, Monogr. Univ. Econ. Innov. Lublin, Innovatio Press Sci. Publ. House Univ. Econ. Innov. Lublin, Lublin, 2012.

[21] H. Grötzsch, Über einige Extremalprobleme der konformen Abbildung. II. Ber. Verh. sächs. Akad. Wiss. Leipzig, Math.-phys. Kl 80 (1928), 497-502. 
[22] H. Guo, Integrable Teichmüller spaces, Sci. China Ser. A 43 (2000), no 1, 47-58.

[23] D. K. Hong and S. G. Rajeev, Universal Teichmller space and Diff $\left(\mathbb{S}^{1}\right) / \mathbb{S}^{1}$. Comm. Math. Phys. 135 (1991), no. 2, $401-411$.

[24] Y. Hu and Y. Shen, On quasisymmetric homeomorphisms. Israel J. Math. 191 (2012), 209-226.

[25] Y.-Z. Huang, Two-dimensional conformal geometry and vertex operator algebras, Progress in Mathematics, vol. 148, Birkhäuser, Boston, MA, 1997.

[26] J. H. Hubbard, Teichmüller theory and applications to geometry, topology, and dynamics. Vol. 1. Teichmüller theory. With contributions by Adrien Douady, William Dunbar, Roland Roeder, Sylvain Bonnot, David Brown, Allen Hatcher, Chris Hruska and Sudeb Mitra. With forewords by William Thurston and Clifford Earle. Matrix Editions, Ithaca, NY, 2006.

[27] J. A. Jenkins, Univalent functions and conformal mapping. Ergebnisse der Mathematik und ihrer Grenzgebiete. Neue Folge, Heft 18.

[28] B. Khesin and R. Wendt, The geometry of infinite-dimensional groups. Ergebnisse der Mathematik und ihrer Grenzgebiete. 3. Folge. A Series of Modern Surveys in Mathematics 51. Springer-Verlag, Berlin, 2009.

[29] A. A. Kirillov and D. V. Yuriev, Representations of the Virasoro algebra by the orbit method, J. Geom. Phys. 5 (1988), no. 3, 351-363.

[30] S. Kushnarev, Teichons: Soliton-like geodesics on universal Teichmüller space. Experiment. Math. 18 (2009), 325-336.

[31] O. Lehto, Univalent functions and Teichmüller spaces, Graduate Texts in Mathematics, Vol. 109, Springer-Verlag, New York, 1987.

[32] I. Markina and A. Vasil'ev, Virasoro algebra and dynamics in the space of univalent functions. Five lectures in complex analysis, Contemp. Math. 525 (2010), 85 - 116, Amer. Math. Soc., Providence, RI.

[33] I. Markina, D. Prokhorov, and A. Vasil'ev, Sub-Riemannian geometry of the coefficients of univalent functions. J. Funct. Anal. 245 (2007), no. 2, 475-492.

[34] S. Nag, The Complex Analytic Theory of Teichmüller Spaces, Canadian Mathematical Society Series of Monographs and Advanced Texts, Wiley, 1988.

[35] S. Nag, A period mapping in universal Teichmller space. Bull. Amer. Math. Soc. (N.S.) 26 (1992), no. $2,280-287$.

[36] S. Nag and D. Sullivan, Teichmüller theory and the universal period mapping via quantum calculus and the $H^{1 / 2}$ space on the circle, Osaka J. Math. 32 (1995), no. 1, 1-34.

[37] S. Nag and A. Verjovsky, Diff( $\left(\mathbb{S}^{1}\right)$ and the Teichmüller spaces, Comm. Math. Phys. 130 (1990), no. 1, $123-138$.

[38] Z. Nehari, Some inequalities in the theory of functions. Trans. Amer. Math. Soc. 75, (1953), 256 - 286.

[39] O. Pekonen, Universal Teichmüller space in geometry and physics, J. Geom. Phys. 15 (1995), no. 3, 227-251.

[40] A. Pfluger, On the Functional $a_{3}-\lambda a_{2}^{2}$ in the class $\mathcal{S}$. Complex Variables 10 (1988), 83-95.

[41] C. Pommerenke, Univalent functions, With a chapter on quadratic differentials by Gerd Jensen. Studia Mathematica/Mathematische Lehrbücher, Band XXV. Vandenhoeck \& Ruprecht, Göttingen 1975.

[42] D. Radnell, Schiffer varation in Teichmüller space, determinant line bundles and modular functors, Ph.D. thesis, Rutgers University, New Bunrswick, NJ, October 2003.

[43] D. Radnell and E. Schippers, Quasisymmetric sewing in rigged Teichmller space, Commun. Contemp. Math. 8 (2006), no. 4, 481-534.

[44] D. Radnell and E. Schippers, A complex structure on the set of quasiconformally extendible nonoverlapping mappings into a Riemann surface, J. Anal. Math., 108 (2009), 277-291.

[45] D. Radnell and E. Schippers, Fiber structure and local coordinates for the Teichmüller space of a bordered Riemann surface, Conform. Geom. Dyn. 14 (2010), 14-34.

[46] D. Radnell and E. Schippers, The semigroup of rigged annuli and the Teichmüller space of the annulus. J. Lond. Math. Soc. (2) 86 (2012), no. 2, $321-342$.

[47] D. Radnell, E. Schippers, and W. Staubach, A Hilbert manifold structure on the Weil-Petersson class Teichmüller space of bordered surfaces, Commun. Contemp. Math. 17 (2015), no. 4, DOI: 10.1142/S0219199715500169. 
[48] D. Radnell, E. Schippers, and W. Staubach, Weil-Petersson class non-overlapping mappings into a Riemann surface, Commun. Contemp. Math. 18 (2016), no. 4. Part of arXiv:1207.0973.

[49] D. Radnell, E. Schippers, and W. Staubach, Dirichlet problem and Sokhotski-Plemelj jump formula on Weil-Petersson class quasidisks, Ann. Acad. Sci. Fenn. Math, 41 (2016), 119-127.

[50] D. Radnell, E. Schippers, and W. Staubach, Quasiconformal maps of bordered Riemann surfaces with $L^{2}$ Beltrami differentials, to appear in J. Anal. Math., Part of arXiv:1403.0868 (2014).

[51] D. Radnell, E. Schippers, and W. Staubach, Convergence of the Weil-Petersson metric on the Teichmüller space of bordered surfaces. Commun. Contemp. Math. 19 (2017). DOI: 10.1142/S0219199716500255. Part of arXiv:1403.0868 (2014).

[52] D. Radnell; E. Schippers; and W. Staubach, Quasiconformal Teichmüller theory as an analytical foundation for two dimensional conformal field theory. To appear in Contemporary Mathematics.

[53] D. Radnell; E. Schippers; and W. Staubach, Dirichlet spaces of domains bounded by quasicircles. Preprint.

[54] K. Reimer and E. Schippers, Grunsky inequalities for mappings into a compact Riemann surface. Complex Analysis and Operator Theory, 9 (2015), no. 8, 1663 - 1679.

[55] A. Schaeffer and D. Spencer, Coefficient regions for schlicht functions. American Mathematical Society Colloquium Publications 35 American Mathematical Society, NY (1950).

[56] M. M. Schiffer, The kernel function of an orthonormal system. Duke Math. J. 13, (1946), $529-540$.

[57] M. M. Schiffer and D. Spencer, Functionals of finite Riemann surfaces. Princeton University Press, Princeton, N. J., 1954.

[58] M. M. Schiffer, Fredholm eigenvalues and conformal mappings. Rend. Mat. e Appl. (5) 22 (1963), 447468.

[59] M. M. Schiffer, Fredholm eigenvalues and Grunsky matrices. Ann. Polon. Math. 39 (1981), 149-164.

[60] M. M. Schiffer, Menahem Max Schiffer: selected papers. Vol. 1. Edited by Peter Duren and Laurence Zalcman. Contemporary Mathematicians. Birkhäuser/Springer, New York, 2013.

[61] M. M. Schiffer, Menahem Max Schiffer: selected papers. Vol. 2. Edited by Peter Duren and Lawrence Zalcman. Contemporary Mathematicians. Birkhuser/Springer, New York, 2014.

[62] E. Schippers, Conformal invariants and higher-order Schwarz lemmas. J. Anal. Math. 90 (2003), 217 241.

[63] E. Schippers, Conformal invariants corresponding to pairs of domains. Future trends in geometric function theory, 207-219, Rep. Univ. Jyväskylä Dep. Math. Stat., 92, Univ. Jyväskylä, Jyväskylä, 2003.

[64] E. Schippers. The power matrix, coadjoint action and quadratic differentials. J. Anal. Math. 98 (2006), 249-277.

[65] E. Schippers, The derivative of the Nehari functional, Annal. Acad. Scient. Fenn. 35 (2010), no. 1, 291-307.

[66] E. Schippers, Conformal invariants corresponding to quadratic differentials. To appear in Israel Journal of Mathematics.

[67] E. Schippers Quadratic differentials and conformal invariants. Journal of Analysis, 24 (2016), 209-228.

[68] H. G. Schmidt, Some examples of the method of quadratic differentials in the theory of univalent functions. Matematisk Institut, Aarhus Universitet, Preprint No. 35 (1970).

[69] M. E. Schonbek, A. N. Todorov, and J. P. Zubelli, Geodesic flows on diffeomorphisms of the circle, Grassmannians, and the geometry of the periodic KdV equation. (English summary) Adv. Theor. Math. Phys. 3 (1999), no. 4, 1027 - 1092.

[70] Y. Shen, On Grunsky operator, Sci. China Ser. A 50 (2007), no. 12, 1805-1817.

[71] Y. Shen, Weil-Petersson Teichmüller space, prepint, arXiv:1304.3197.

[72] L. Takhtajan and L.-P. Teo, Weil-Petersson Metric on the Universal Teichmüller Space. Memoirs of the American Mathematical Society. 183 (2006), no. 861.

[73] O. Tammi, Extremum problems for bounded univalent functions. I. Lecture Notes in Mathematics, 646, Springer-Verlag, Berlin-New York, 1978.

[74] O. Tammi, Extremum problems for bounded univalent functions. II. Lecture Notes in Mathematics, 913, Springer-Verlag, Berlin-New York, 1982.

[75] S. Tang, Some characterizations of the integrable Teichmller space. Sci. China Math. 56 (2013), no. 3, $541-551$. 
[76] O. Teichmüller, Ungleichungen zwischen den Koeffizienten schlichter Funktionen. Stzgsber. preuss. Akad. Wiss., Math.-naturwiss. Kl. (1938) 363-375.

[77] A. Vasil'ev, Moduli of families of curves for conformal and quasiconformal mappings. Lecture Notes in Mathematics, 1788. Springer-Verlag, Berlin, 2002.

[78] S. Wolpert, Chern forms and the Riemann tensor for the moduli space of curves. Inv. Math. 85 (1), (1986), 119-145.

[79] M. Yanagishita, Introduction of a complex structure on the p-integrable Teichmüller space. Ann. Acad. Sci. Fenn. Math. 39 (2014), no. 2, 947 - 971.

[80] M. Yanagishita, Kählerity and negativity of Weil-Petersson metric on square integrable Teichmüller space. J. Geom. Anal. 27 (2017), no. 3, 1995-2017.

Eric Schippers, Department of Mathematics, University of Manitoba, Winnipeg, ManiTOBA, R3T 2N2, CANADA

E-mail address: eric_schippers@umanitoba.ca

Wolfgang Staubach, Department of Mathematics, Uppsala University, Box 480, 75106 UpPSAla, Sweden

E-mail address: wulf@math.uu.se 